\title{
Identificação de relações entre Melhoria Contínua e Inovação de produtos e processos por meio de revisão bibliográfica sistemática
}

\section{Identification of relations between Continuous Improvement and Innovation of products and processes: a systematic literature review}

\author{
Fabiane Letícia Lizarelli' \\ José Carlos de Toledo'
}

\begin{abstract}
Resumo: A Melhoria Contínua (MC) e a Inovação de produtos e processos são estratégias que geram vantagem competitiva para as organizações, seja em relação ao custo ou à diferenciação. A MC e a Inovação precisam ser gerenciadas de forma a assegurar, no longo prazo, os resultados desejados. Na literatura, são observadas afirmações recorrentes sobre a necessidade de coexistência da MC e da Inovação e de que estas devem estar alinhadas e ser conduzidas de forma colaborativa e complementar. Porém, existe uma lacuna teórica sobre quais são as relações existentes, no âmbito das organizações, que possibilitam a interação e a colaboração desejadas entre os projetos e as pessoas dessas duas áreas. Com o intuito de identificar e analisar essas relações foi realizada uma Revisão Bibliográfica Sistemática da literatura que aponta conexões entre atividades de MC e de Inovação de produtos e processos. Com base nos artigos revisados, foi possível esquematizar as principais relações e apontar as principais práticas gerenciais que as sustentam. Os resultados mostraram que as publicações apresentam relações de escopo bastante diferenciado, porém estas não são devidamente exploradas e desdobradas, sendo possível identificar lacunas para pesquisas futuras.

Palavras-chave: Revisão bibliográfica sistemática; Melhoria Contínua; Inovação; Relações entre MC e Inovação.
\end{abstract}

\begin{abstract}
Continuous Improvement (CI) and Innovation of products and processes generate competitive advantage for organizations, whether in relation to the cost or differentiation. Both CI and Innovation must be managed to ensure the desired results. The literature points out the necessity of coexistence of CI and Innovation, and the authors affirm that these should be aligned and developing a collaborative role. However, there is a theoretical gap about which relationships enable the desired interaction and collaboration between CI and Innovation. A Systematic Literature Review was used in order to identify and analyze the relations that specifically demonstrate connections between CI and Innovation. Based on the articles reviewed, it was possible to lay out the main relationships, pointing out the main management practices that sustain each relationship and other relevant information. The results showed relationships of different scopes, but these are not widely exploited, and gaps were identified for future research.
\end{abstract}

Keywords: Systematic literature review; Continuous Improvement; Innovation; Relationships between Continuous Improvement and Innovation.

\section{Introdução}

A Melhoria Contínua (MC) possui estreita relação com a Inovação de produtos e processos. Além da introdução de novos produtos e processos, os consumidores e o mercado exigem que esses possuam níveis de desempenho cada vez melhores, o que pode ser obtido com o uso de programas de MC (Toledo, 1994; Mesquita \& Alliprandini, 2003; Fryer et al., 2007). A compreensão e o estudo das relações entre esses dois temas são de fundamental importância para as organizações (Irani et al., 2004).
A MC pode ser compreendida como um processo de mudança incremental, contínua, abrangendo todas as pessoas da empresa (Caffyn, 1999). O foco da mudança pode ser em produtos, processos produtivos (Imai; 1992; McAdam \& Armstrong, 2001; Garcia \& Calantone, 2002) e processos organizacionais (MacDonald, 1995; Tonnessen, 2005). O foco da Inovação, segundo a OCDE (2005), pode ser em produtos e processos, métodos de marketing e organizacionais. Assim, pode-se considerar que há diversos objetos semelhantes para as ações de Inovação

${ }^{1}$ Departamento de Engenharia de Produção, Universidade Federal de São Carlos - UFSCar, Rod. Washington Luís, Km 235, SP-310, CEP 13565-905, São Carlos, SP, Brasil, e-mail: fabiane@dep.ufscar.br; toledo@dep.ufscar.br

Recebido em Jan. 30, 2014 - Aceito em Maio 8, 2015

Suporte financeiro: Nenhum. 
e de $\mathrm{MC}$, como produtos processos produtivos e organizacionais.

AMC e a Inovação, tanto de produto como de processo, podem ser consideradas funções complementares em diversas organizações, mas, em geral, são executadas por equipes e áreas funcionais distintas (Imai, 1992), além de diferirem também em relação aos objetivos, nível de novo conhecimento utilizado e práticas de gestão (McAdam et al., 1998; Prajogo \& Sohal, 2001). Para que haja colaboração entre essas atividades, há a necessidade de um gerenciamento interfuncional, para possibilitar integração e contribuição conjunta para os objetivos competitivos da empresa.

Não há consenso sobre as vantagens da coexistência e colaboração entre atividades de MC e de Inovação. Para alguns autores, a estrutura e as práticas geradas pela MC podem dificultar a introdução de inovações abruptas e as estruturas gerenciais e organizacionais necessárias para que haja relações entre os dois temas podem ser complexas e difíceis de serem obtidas (Adler et al., 1999; Lam, 2004; Prajogo \& Sohal, 2001; Cole \& Matsumiya, 2008). Em organizações em contextos em que a competitividade depende mais de mudanças abruptas, a abordagem da MC e suas relações com a Inovação podem não contribuir para a competitividade (Prajogo \& Sohal, 2001; Cole \& Matsumiya, 2008; Mirica \& Ito, 2010), já que a MC é focada no aumento da eficiência dentro dos limites de uma mesma trajetória tecnológica e de padrões de conhecimento existentes (Benner \& Tushman, 2003). Além disso, a forte cultura de melhoria e o foco em mudanças incrementais podem levar a empresa a ter dificuldades com mudanças maiores de tecnologia e de mercado (Benner \& Tushman, 2003; Prajogo \& Sohal, 2001; Cole \& Matsumiya, 2008; Mirica \& Ito, 2010).

Outra visão é de que a coexistência entre os dois temas é positiva e gera melhores resultados quanto ao desempenho de produtos e de processos (Tushman \& O'Reilly, 1996; Thamhain, 2003; Tidd et al., 2008). As estruturas organizacionais para relacionar os dois temas e os tipos de relações podem ser diversos e ainda não estão claras na literatura quais são as possíveis formas de conexão, interação e de colaboração entre os projetos e atividades e como alcançá-las (Boer \& Gertsen, 2003; Chapman \& Corso, 2005; Mirica \& Ito, 2010; Corso \& Gastaldi, 2011).

A maior ou menor possibilidade de relações entre esses dois temas (áreas) é condicionada por fatores como o setor em que a empresa está inserida, as necessidades do mercado e a trajetória tecnológica dos produtos e processos.

Prajogo \& Sohal (2001) afirmam que a discussão sobre as relações entre MC e Inovação é importante porque argumentos conflitantes aparecem na literatura, além de não ser uma discussão frequente (Perdomo-Ortiz et al., 2006; Martínez-Costa \& Martínez-Lorente, 2008). É perceptível que existe uma lacuna de conhecimento que precisa ser preenchida (Perdomo-Ortiz et al., 2006).

Como esses temas são de interesse tanto da área acadêmica quanto empresarial, é importante estudar não apenas seus escopos, mas também as possíveis inter-relações e complementaridades entre eles (McAdam et al., 1998; Perdomo-Ortiz et al., 2006).

O desenvolvimento de um novo produto, considerado aqui como uma inovação, pode ser uma oportunidade para que se realizem melhorias nos processos nos quais o novo produto será manufaturado (Corso \& Pavesi, 2000; Chapman \& Hyland, 2004). Desenvolvimentos de novos produtos podem auxiliar, também, na geração de melhorias de produtos existentes, visto que é um momento de captação de ideias e de soluções (Chapman \& Hyland, 2004; Nilsson-Witell et al., 2005).

As atividades de Inovação podem gerar possibilidades de futuros projetos de melhoria, tanto para aperfeiçoamento da inovação gerada, quanto para a melhoria de produtos já existentes e, para isso, deve haver conexão e interação entre as pessoas e atividades de MC e de Inovação (Hoerl \& Gardner, 2010).

Por outro lado, as atividades de Melhoria Contínua podem apoiar e gerar inputs para as atividades de Inovação (Irani \& Sharp, 1997; Terziovski, 2002; Benner \& Tushman, 2003; Tidd et al., 2008; Martínez-Costa \& Martínez-Lorente, 2008; Anand et al., 2009).

Considerando a perspectiva que reconhece vantagens nessas relações e na sua gestão, justifica-se a importância de compreender quais interações podem e devem haver entre MC e Inovação. A presença das supostas relações é dependente do estímulo a práticas, ou seja, a comportamentos habituais na empresa que possibilitam que a relação seja desenvolvida de forma mais sólida.

O objetivo deste artigo é identificar e analisar as relações, e suas práticas, entre MC e Inovação de produtos e processos presentes na literatura, gerando conhecimentos para estudos posteriores. As relações podem ser geradas tanto a partir dos projetos e pessoas relacionados à $\mathrm{MC}$ em direção aos projetos e atividades de Inovação, quanto no sentido oposto.

O método de pesquisa utilizado para a investigação é a Revisão Bibliográfica Sistemática (RBS). Por meio da RBS foi possível identificar as relações mais recorrentes, as práticas associadas e propor uma estrutura conceitual para as principais relações entre MC e Inovação.

\section{Conceituação e diferenciação entre MC e Inovação}

De acordo com Prajogo \& Sohal (2001), existem várias definições de Inovação, ainda que estas não sejam excludentes. Inovação se refere a fazer algo novo, e é diferente de invenção ou criatividade, pois deve gerar algum impacto econômico para a 
organização. Consiste na introdução de novos produtos e métodos produtivos, na abertura de novos mercados, na descoberta de matérias-primas e na implantação de novas formas de organização (Schumpeter, 1934 apud Rejeb et al., 2008).

Para Tidd et al. (2008), Inovação representa mudança, podendo-se descrevê-la em quatro categorias: Inovação de produto, que é a mudança nos produtos/serviços que uma empresa oferece; Inovação de processo, que é a mudança na forma que tais produtos/serviços são produzidos e entregues; Inovação de posição, que é a mudança no contexto de inserção de mercado desses produtos/serviços; e, Inovação de paradigma, que é a mudança nos modelos mentais que orientam o que a empresa faz.

Neste trabalho, adota-se a definição de Inovação do Manual de Oslo, segundo o qual a Inovação pode englobar a implantação de um produto (bem ou serviço) novo ou significantemente melhorado, de um processo, de um novo método de marketing, ou de um novo método organizacional (OCDE, 2005).

Inovações de produtos e de processos são chamadas de inovação tecnológica, que pode ser compreendida como a introdução de produtos ou métodos produtivos novos ou melhorados, que adicionam valor econômico à empresa (Abernathy \& Utterback, 1978; Ettlie et al., 1984; Schumpeter, 1985; Damanpour, 1987; Zairi, 1995; Sciulli, 1998; Garcia \& Calantone, 2002; Tidd et al., 2008).

A inovação tecnológica pode ser incremental ou radical, considerando-se características como o nível da mudança (menor versus maior), o mercado ou o consumidor-alvo (existente versus novo) e o nível do risco da inovação (baixo versus alto) (Kim et al., 2012).

Inovação radical incorpora novos conhecimentos e tecnologias que representam uma ruptura nas práticas e competências existentes. Enquanto a inovação incremental representa aperfeiçoamentos na trajetória tecnológica existente, realizados com a capacidade técnica corrente da organização (Dewar \& Dutton, 1986; Benner \& Tushman, 2003; Grover et al., 2007).

Considerando um continuum desde a inovação incremental até a inovação radical, existem dúvidas sobre onde a MC estaria posicionada, já que também trata de mudanças tecnológicas em produtos e processos.

Jha et al. (1996) definem MC como um conjunto de atividades que constituem um processo de raciocínio e intervenção que busca alcançar a melhoria de desempenho. Bhuiyan et al. (2006) definem MC como uma cultura de melhoria sustentável, visando, por meio do envolvimento de todos os participantes da organização, eliminar desperdícios em todos os sistemas e processos organizacionais. Trata-se de todos trabalhando juntos para melhorar, com foco nas metas organizacionais e sem, necessariamente, grandes investimentos de capital (Bessant \& Francis, 1999; Bhuiyan \& Baghel, 2005).
De acordo com Imai (1992), uma das principais diferenças entre a Inovação e a MC é a constância das mudanças. A MC tem o objetivo de ser gradual, sistemática (Imai, 1992; Bessant et al., 1994; Bessant \& Francis, 1999; Caffyn, 1999; Savolainen, 1999; Bhuiyan et al., 2006); com foco nas pessoas e no envolvimento do trabalho em grupo (Imai, 1992; Jha et al., 1996; Andersson et al., 2006); com esforços para melhores resultados de eficiência de produtos e processos já existentes (Bhuiyan et al., 2006); conduzida de forma incremental e ininterrupta (Imai, 1992). A Inovação tem um caráter mais intermitente (Imai, 1992; Leifer et al., 2000; McLaughlin et al., 2008); com um processo de gestão mais complexo (McLaughlin et al., 2008); possibilidade de uso de novas tecnologias e mudança de trajetória tecnológica (Henderson \& Clark, 1990; Imai, 1992; Corso \& Pellegrini, 2007); geralmente envolvendo grandes investimentos e esforços para aquisição de conhecimento (Imai, 1992; Tidd et al., 2008).

Do ponto de vista da adição de valor, para alguns autores, as atividades e projetos englobados pela MC podem ser considerados como inovação incremental (Caffyn \& Bessant, 1996), pois a MC visa a melhoria de índices de desempenho de produtos e processos (Imai, 1992).

Para Caffyn \& Bessant (1996) e Caffyn (1999), a MC envolve atividades de inovação incremental, como resposta às necessidades de clientes e às mudanças do mercado, abrangendo toda a empresa, no sentido de aproveitar e estimular as ideias de todos. Bessant et al. (1996) e Tidd et al. (2008) classificam os projetos e atividades de MC como inovação de alto envolvimento, pois estimulam as pessoas a encontrarem novas soluções com o objetivo de melhoria de desempenho, característicos da inovação incremental.

Por outro lado, os teóricos relacionados à Inovação preferem limitar a inovação em termos de mudança radical e a distinguem da mudança incremental, que preferem chamar de melhoria (Abernathy \& Utterback, 1978). Geralmente argumenta-se que a melhoria é, simplesmente, "fazer algo já existente de uma maneira melhor", enquanto a inovação é "fazer algo diferente" (McAdam et al., 1998; Prajogo \& Sohal, 2001).

Apesar de alguns autores considerarem que a MC pode ser focada na inovação incremental (Bessant et al., 1996; Caffyn \& Bessant, 1996; Bessant \& Francis, 1999; Tidd et al., 2008) e outros não considerarem a mudança incremental como um aspecto da Inovação, sendo considerada apenas melhoria (Abernathy \& Utterback, 1978), a principal diferenciação entre Inovação e MC é que esta é um processo que permite que as pessoas, geralmente em equipes, possam focar seu pensamento e ações para melhorar continuamente, de forma ininterrupta, práticas, 
processos, sistemas, produtos e/ou serviços já existentes (Imai, 1992; Jha et al., 1996; Bhuiyan et al., 2006).

$\mathrm{O}$ conceito de inovação incremental, mesmo que mais próximo do conceito de $\mathrm{MC}$, representa aperfeiçoamentos na trajetória tecnológica existente, realizados com a capacidade técnica corrente (Dewar \& Dutton, 1986; Benner \& Tushman, 2003; Grover et al., 2007). Portanto, a inovação incremental não apresenta a questão de continuidade exigida pela MC e apresenta um caráter de novidade ainda que não seja uma mudança abrupta, de ruptura, revolucionária, como a inovação radical (Damanpour \& Gopalakrishnan, 1998; Garcia \& Calantone, 2002; McLaughlin et al., 2008).

De acordo com Bhuiyan \& Baghel (2005) e Andersson et al. (2006), a MC é disseminada pela organização por meio de programas e metodologias. Os mais utilizados pelas organizações são: a Gestão da Qualidade Total, em que a MC é a forma de obtenção de melhores níveis de desempenho de produtos e processos em relação à qualidade com foco no cliente; o programa Seis Sigma que visa a redução da variabilidade e erro por meio da $\mathrm{MC}$; o Kaizen, relacionado com o Lean Production, que visa a participação das pessoas com foco na diminuição de desperdícios; e o Lean-Sigma, que trabalha conjuntamente as técnicas, ferramentas e objetivos das abordagens Lean e Seis Sigma em relação à melhoria de produtos e processos (Andersson et al., 2006; Bhuiyan et al., 2006).

Os projetos, atividades, objetivos e impactos da $\mathrm{MC}$ variam de acordo com os programas em que estão inseridos, com o alinhamento com a estratégia da empresa e com o nível de maturidade da MC (Oprime \& Lizarelli, 2010; Zampini \& Toledo, 2010). Os projetos Seis Sigma são conduzidos em diversas áreas organizacionais e com diferentes níveis de complexidade e de mudanças relacionados à estratégia da empresa (Andersson et al., 2006). Os projetos de MC pautados pela abordagem Lean focam, principalmente, melhorias de velocidade, eficiência e fluxo de processos. Já no TQM (Total Quality Management), é enfatizado o envolvimento de todos os colaboradores, com o objetivo de melhorar e padronizar processos (Andersson et al., 2006).

Assim, o nível de mudança das ações de MC pode variar de empresa para empresa e em função dos programas implantados. Portanto, o nível das mudanças geradas pela MC pode variar desde a introdução de padronização até a realização de mudanças incrementais nos padrões existentes. A padronização engloba as ações de MC que envolvem sistematização e controle e que não geram novas soluções para produtos e processos.

A Figura 1 apresenta um continuum englobando as atividades e projetos de MC e de Inovação, baseado na literatura consultada, o qual será base para a discussão ao longo deste artigo.

\section{Procedimento de pesquisa bibliográfica}

O foco da pesquisa é a identificação das relações entre MC e Inovação e das respectivas práticas. O termo "relação" é compreendido como a forma como os dois temas estão conectados ou ligados, o estado de estar conectado (Oxford University, 2013; Cambridge, 2013). Assim, neste trabalho, "relação" é definida como as várias formas de colaboração, conexão, ligação e interação entre Melhoria Contínua e Inovação.

Para a existência efetiva de cada relação na organização, práticas específicas devem ser incentivadas. O termo "prática" pode ser entendido como a forma habitual de funcionamento ou comportamento (WordNet ${ }^{\circledR}, 2013$ ). Assim, neste trabalho, as práticas são consideradas como os comportamentos habituais que contribuem para a existência das relações entre MC e Inovação.

O procedimento técnico utilizado para a realização da pesquisa foi a Revisão Bibliográfica Sistemática

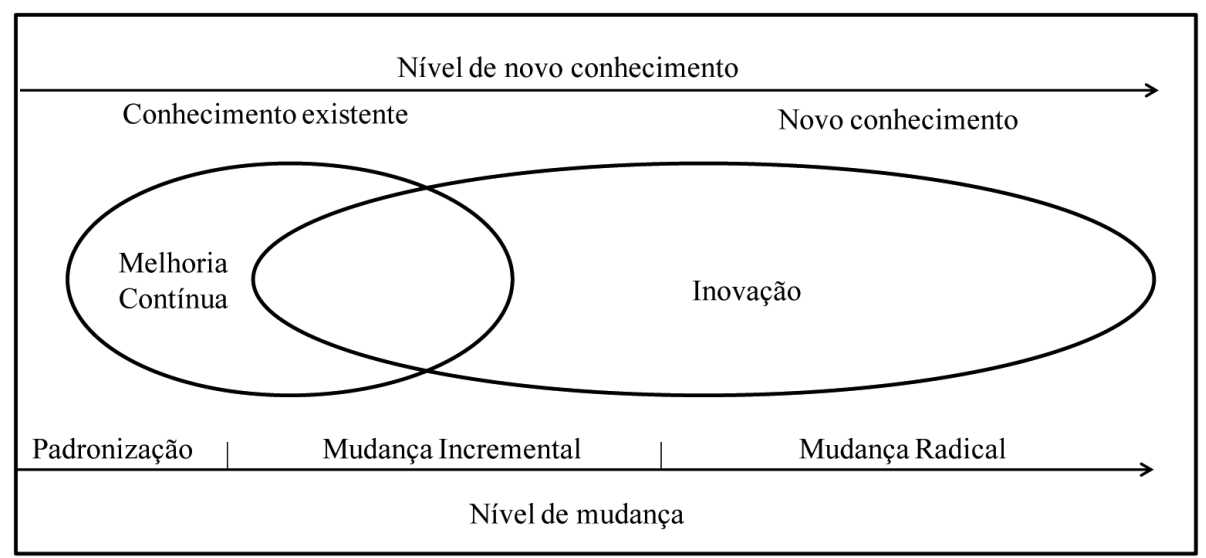

Figura 1. Continuum englobando atividades de MC e Inovação. Fonte: Elaborado pelo autor. 
(RBS). A RBS utiliza uma metodologia bem definida para identificar, analisar e interpretar todas as evidências disponíveis relacionadas a uma questão de pesquisa específica, ou fenômeno de interesse de uma forma que é imparcial, reproduzível e baseada em evidências (Tranfield et al., 2003; Levy \& Ellis, 2006; Kitchenham \& Charters, 2007).

Para a condução da RBS, utilizaram-se as recomendações de Biolchini et al. (2007), em que as etapas principais são: planejamento, execução e análise (Figura 2). Brereton et al. (2007) salientam a importância do planejamento, que consiste na elaboração e validação de um protocolo para a realização da pesquisa. O protocolo envolve a definição do problema da revisão, da questão que deve ser respondida, das fontes e bases de dados que serão utilizadas, dos idiomas das publicações, das palavras-chave mais adequadas para a busca e dos critérios de inclusão e exclusão da publicação para a RBS.

A pergunta a ser respondida pela RBS foi: quais são as relações existentes entre MC e Inovação e quais práticas podem efetivá-las?

As bases de dados eletrônicas selecionadas como fonte de buscas foram: ISI Web of Knowledge, Science
Direct, Scopus e Engineering Village. Essas bases foram escolhidas por apresentarem grande número de periódicos, conferências e artigos de interesse para a área de estudo e abrangência internacional na área de pesquisa. As bases foram acessadas no período de janeiro a abril de 2013 .

A busca considerou os últimos 25 anos de publicação (1989 a início de 2013). Os idiomas selecionados foram: o inglês, pois é o idioma universalmente aceito para redação de trabalhos científicos e encontrado em todas as bases de dados pesquisadas; o português; e o espanhol.

A execução da RBS foi constituída de seis etapas. $\mathrm{Na}$ etapa 1 , as buscas foram realizadas, observando-se no título dos trabalhos, os termos "continuous improvement" ou "kaizen" somados a "innovation" ou "product development" ou "process development". Porém, esta primeira busca retornou poucos resultados (Tabela 1). A busca foi expandida para o resumo e palavras-chave (etapa 2), utilizando os mesmos termos de busca, retornando um número maior de resultados (Tabela 1).

A terceira etapa constituiu o uso de filtros para a seleção de resultados pertinentes, focando as áreas de

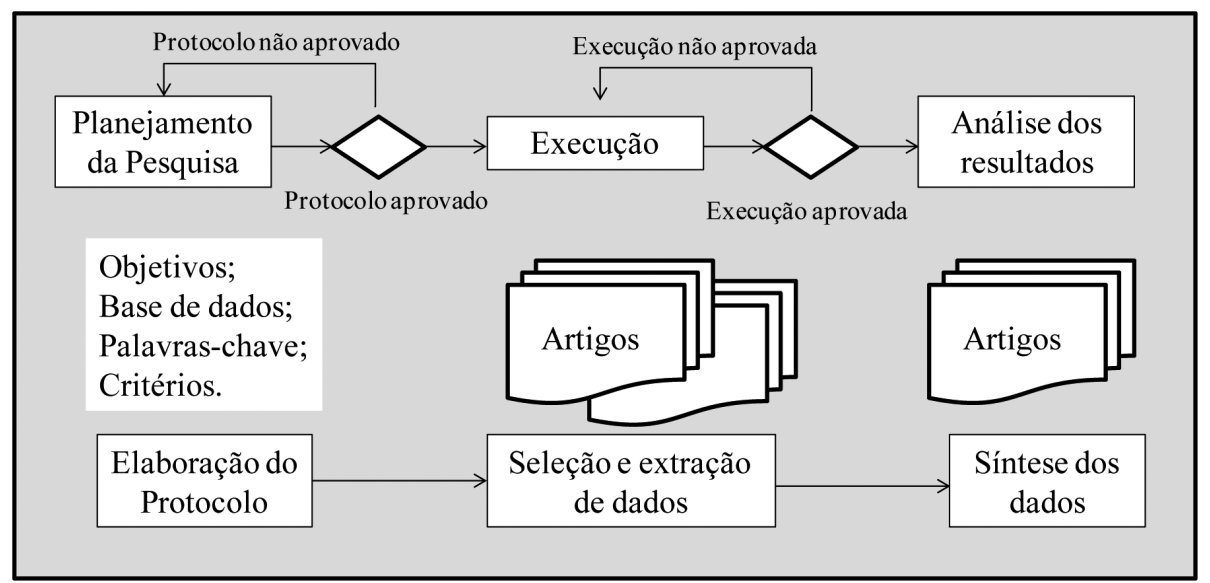

Figura 2. Condução da revisão bibliográfica sistemática. Fonte: Biolchini et al. (2007).

Tabela 1. Resultados, em número de publicações, para a RBS.

\begin{tabular}{|c|c|c|c|c|c|c|}
\hline \multirow[b]{2}{*}{$\begin{array}{c}\text { Base de } \\
\text { periódicos }\end{array}$} & \multicolumn{6}{|c|}{ Etapas de busca e seleção } \\
\hline & $\begin{array}{l}1 \text { - Busca } \\
\text { apenas no } \\
\text { título }\end{array}$ & $\begin{array}{l}2 \text { - Busca no } \\
\text { títlulo mais } \\
\text { palavras- } \\
\text { chave e } \\
\text { resumo }\end{array}$ & $\begin{array}{l}\text { 3- Filtros de } \\
\text { áreas, línguas } \\
\text { e fontes }\end{array}$ & $\begin{array}{c}4 \text { - Leitura de } \\
\text { título, resumo } \\
\text { e verificação da } \\
\text { disponibilidade }\end{array}$ & $\begin{array}{c}5 \text { - Seleção } \\
\text { após leitura } \\
\text { completa }\end{array}$ & $\begin{array}{l}\text { 6- Eliminação } \\
\text { de artigos } \\
\text { repetidos nas } \\
\text { bases }\end{array}$ \\
\hline $\begin{array}{l}\text { Engineering } \\
\text { Village }\end{array}$ & 20 & 453 & 320 & 61 & 29 & 29 \\
\hline $\begin{array}{l}\text { ISI Web of } \\
\text { Knowledge }\end{array}$ & 24 & 313 & 172 & 55 & 30 & 3 \\
\hline Science Direct & 5 & 54 & 54 & 46 & 16 & 16 \\
\hline Scopus & 34 & 840 & 511 & 41 & 15 & 6 \\
\hline Total & 83 & 1660 & 1057 & 203 & 90 & 54 \\
\hline
\end{tabular}

Fonte: Elaborado pelo autor. 
business, management, social sciences e engineering, ou similares, em cada base. Excluíram-se também resultados de outros idiomas que não os selecionados e documentos como patentes e livros, mantendo principalmente arquivos de congressos e periódicos.

Depois do primeiro filtro, todos os títulos e resumos foram lidos, e foram selecionados os artigos que atendiam à pergunta principal da RBS e abordavam alguma relação entre MC e Inovação (etapa 4). Os arquivos selecionados e que estavam disponíveis nas bases de dados foram baixados. Os que não estavam disponíveis tiveram seu título e autores colocados no Google acadêmico para verificar se não haveria alguma outra fonte para leitura, ou solicitado aos autores o envio do artigo por e-mail.

Foi realizada a leitura completa dos artigos baixados, e os que atenderam ao critério "identificação e ou recomendação de relações, ações conjuntas e gestão integrada entre MC e Inovação" foram selecionados para a análise e compilação das informações (etapa 5). Depois da identificação de uma relação, buscaram-se no artigo as práticas associadas.

A última etapa (6) foi constituída da retirada de artigos repetidos nas diversas bases. Como a análise iniciou-se pelo Engineering Village e Science
Direct, estas bases têm um maior número de artigos selecionados.

Para as 54 publicações selecionadas, as seguintes informações foram registradas: título da publicação; autores; ano da publicação; nome do periódico ou conferência; bases em que foram encontradas; número de citações do artigo (plataforma Google acadêmico e ISI Web of Knowledge), método de pesquisa empregado e identificação de relações e práticas. As informações podem ser observadas no Quadro 1, no qual são apresentados dois exemplos para as informações captadas.

Depois da coleta das informações, foi realizada a identificação das principais relações entre $\mathrm{MC}$ e Inovação, as quais foram agrupadas por semelhança. Uma nova leitura foi realizada de todas as publicações, para verificar se haveria alguma outra relação presente que não havia sido percebida anteriormente. A planilha construída para a avaliação das relações pode ser observada, parcialmente, no Quadro 2. Este quadro mostra um exemplo da análise de 3 das relações encontradas em 3 dos artigos analisados.

Com a análise das 54 publicações de periódicos e congressos, foram identificadas dezesseis tipos de relações. Outros resultados podem ser observados no próximo tópico deste artigo.

Quadro 1. Informações sobre as publicações selecionadas.

\begin{tabular}{|c|c|c|}
\hline Informações & Artigo 1 & Artigo 2 \\
\hline Título & $\begin{array}{l}\text { How companies are reinventing their } \\
\text { idea-to-launch methodologies }\end{array}$ & $\begin{array}{l}\text { Perspective: The Stage-Gates Idea-to-Launch } \\
\text { Process-Update, What's New, and Nex Gen } \\
\text { Systems }\end{array}$ \\
\hline Autores & Robert Cooper & Robert Cooper \\
\hline Fonte/Data & $\begin{array}{l}\text { Technology Management } \\
\text { March, April 2009, Vol. 52, No. 2, p. 47-57 }\end{array}$ & $\begin{array}{l}\text { Journal of Product Innovation Management, } \\
\text { 2008, Vol. 25, p. 213-232 }\end{array}$ \\
\hline Bases & Web of Science e Scopus & Web of Science e Scopus \\
\hline $\mathrm{N}^{\mathrm{o}}$ de citações & $\begin{array}{l}\text { Google Acadêmico (67) } \\
\text { ISI Web of Knowledge (17) }\end{array}$ & $\begin{array}{l}\text { Google Acadêmico (410) } \\
\text { ISI Web of Knowledge (90) }\end{array}$ \\
\hline $\begin{array}{l}\text { Método de pesquisa } \\
\text { do artigo }\end{array}$ & Revisão Bibliográfica & Revisão Bibliográfica \\
\hline $\begin{array}{l}\text { Relações entre MC } \\
\text { e Inovação }\end{array}$ & $\begin{array}{l}\text { - A MC é utilizada para a melhoria do } \\
\text { processo de desenvolvimento de novos } \\
\text { produtos (PDP). } \\
\text { - Deve ser instituída a cultura da MC no pós- } \\
\text { lançamento dos produtos. }\end{array}$ & $\begin{array}{l}\text { - Aplicação de ferramentas das abordagens } \\
\text { Lean e Seis Sigma para a melhoria do PDP. } \\
\text { - Aplicação da cultura de MC após a fase de } \\
\text { lançamento dos produtos. }\end{array}$ \\
\hline Práticas & $\begin{array}{l}\text { - Utilizar ferramentas e técnicas da MC, } \\
\text { como o Mapa de Fluxo de Valor, para a } \\
\text { identificação e eliminação de desperdícios } \\
\text { no PDP. } \\
\text { - Analisar as causas de problemas no PDP. } \\
\text { - Não culpar equipe do PDP, quando } \\
\text { problemas ocorrem. } \\
\text { - Aplicar métricas de desempenho no PDP } \\
\text { visando identificar possibilidades de } \\
\text { melhorias e aplicá-las. }\end{array}$ & $\begin{array}{l}\text { - Utilizar ferramentas e técnicas da MC, } \\
\text { como o Mapa de Fluxo de Valor, para a } \\
\text { identificação e eliminação de desperdícios } \\
\text { no PDP. } \\
\text { - Analisar as causas de problemas no PDP. } \\
\text { - Não culpar equipe do PDP, quando } \\
\text { problemas ocorrem. } \\
\text { - Aplicar métricas de desempenho no PDP } \\
\text { visando identificar possibilidades de } \\
\text { melhorias e aplicá-las. }\end{array}$ \\
\hline
\end{tabular}

Fonte: Elaborado pelo autor. 
Quadro 2. Presença das relações em cada um dos artigos selecionados.

\begin{tabular}{|l|c|c|c|}
\hline Relações entre MC e Inovação & \multicolumn{2}{|c|}{ Artigos } \\
\hline & $\begin{array}{c}\text { How companies } \\
\text { are reinventing } \\
\text { their idea-to-launch } \\
\text { methodologies - Cooper } \\
\mathbf{( 2 0 0 9 )}\end{array}$ & $\begin{array}{c}\text { The Stage-Gates } \\
\text { Idea-to-Launch } \\
\text { Process-Update, } \\
\text { What's New, and } \\
\text { NexGen Systems - } \\
\text { Cooper (2008) }\end{array}$ & $\begin{array}{c}\text { Continuous product } \\
\text { innovation: A } \\
\text { comparison of key } \\
\text { elements across different } \\
\text { contingency sets - } \\
\text { Chapman et al. (2001) }\end{array}$ \\
\hline $\begin{array}{l}\text { Geração de projetos ou ações de } \\
\text { melhoria do produto alinhadas à } \\
\text { inovação de produtos }\end{array}$ & Ausente & Ausente & Presente \\
\hline $\begin{array}{l}\text { Geração de projetos ou ações de } \\
\text { melhoria do processo alinhadas à } \\
\text { inovação de produtos }\end{array}$ & Ausente & Ausente & Presente \\
\hline $\begin{array}{l}\text { Utilização de práticas e } \\
\text { ferramentas de MC na gestão de } \\
\text { processo e projetos da área de } \\
\text { Pesquisa \& Desenvolvimento \& } \\
\text { Inovação, incluindo o PDP }\end{array}$ & Presente & Presente & Ausente \\
\hline
\end{tabular}

Fonte: Elaborado pelo autor.

\section{Apresentação e discussão dos resultados da RBS}

Em relação ao ano de publicação, os resultados encontrados para os artigos que apresentavam uma ou mais relações entre $\mathrm{MC}$ e Inovação podem ser observados na Figura 3. O artigo mais antigo é de 1991. O número médio de trabalhos publicados cresceu nos últimos quatro anos (2009-2012), porém existem sazonalidades. Em 2013, aparece apenas uma publicação porque apenas dois meses do ano foram observados.

As maiores incidências de publicação mostram algumas tendências de pesquisa ao longo do tempo, por exemplo, os artigos de 1991-1997 focavam principalmente na estruturação da $\mathrm{MC}$ e sua relação com a gestão da Inovação, auxiliando na implantação de uma cultura de Inovação. Os artigos do início dos anos 2000 focam a evolução do nível de maturidade da MC e a aplicação da filosofia da MC para a inovação contínua de produtos. Os artigos mais recentes evidenciam principalmente a preocupação com a inserção de práticas de melhoria para melhor gestão de projetos de Pesquisa e Desenvolvimento, incluindo o Processo de Desenvolvimento de Produtos.

Nas 54 publicações há 124 diferentes autores. Dos autores identificados, 22 apresentam autoria em mais de um artigo (Figura 4). Os principais autores são: Bessant, J.; Corso, M.; Caffyn, S; Irani, Z., os quais apresentam maior número de publicações. Esses quatro primeiros autores são responsáveis por $27,8 \%$ das publicações selecionadas ( 15 das 54 estão sob autoria de um ou mais deles), mostrando grande concentração de pesquisadores que estudam o tema. Os autores indicados na Figura 4 (os 22 principais), são responsáveis por $51,85 \%$ das publicações selecionadas.
Em relação à fonte, $12,96 \%$ das publicações são provenientes de anais de congressos, os outros $87,04 \%$ são publicações de periódicos. Os periódicos e congressos contendo mais publicações podem ser observados na Tabela 2. A revista Technovation é a principal fonte, contendo $18,52 \%$ das publicações analisadas, seguida da International Journal of Technology Management, com 7,41\% das publicações selecionadas. Assim, esses dois periódicos representam oportunidades para pesquisadores da área submeterem seus artigos e terem avaliações de especialistas.

Para análise das principais publicações, foi realizado o levantamento do Fator de Impacto do JCR (Journal Citation Reports), do ano de 2012. É possível observar na Figura 5 que a revista Technovation tem o terceiro maior fator de impacto, e 10 dos 54 artigos (18,52\%) analisados foram publicados nesta revista. Foi encontrado o fator de impacto apenas de 14 periódicos, o que compreendeu 26 publicações. A Figura 5 apresenta o número de publicações das cinco revistas com maior JCR.

Nas Figuras 6 e 7, podem ser visualizadas as publicações mais citadas das 54 utilizadas, com base, respectivamente, nos dados do Google acadêmico e do ISI WEB of Knowledge. O Google acadêmico foi utilizado porque nem todas as publicações estavam disponíveis na plataforma ISI. Mas as dez primeiras mais citadas no Google acadêmico, com exceção do artigo de Ayas (1996), também estavam na plataforma ISI, por esse motivo houve apenas a exclusão deste artigo e a inserção do artigo de Yang et al. (2010) na Figura 7. Essas podem ser consideradas as principais publicações analisadas, considerando o critério do número de citações. 
Pela análise das duas fontes de número de citações, pode-se perceber que o artigo de Cooper (2008), focado nas formas de melhoria do processo de Desenvolvimento de Produtos, é o mais citado. Os próximos 3 artigos mais citados, nas duas fontes, junto com o artigo de Bessant et al. (1996), são relacionados aos comportamentos e aos níveis de maturidade da organização em MC. Os artigos de Bartezzaghi et al. (1997) e de Chapman \& Hyland (2004) estão relacionados com a inovação contínua do produto, focando as contribuições das ações de MC para as inovações de produtos.

As relações entre MC e Inovação, identificadas nas 54 publicações analisadas, foram agrupadas em

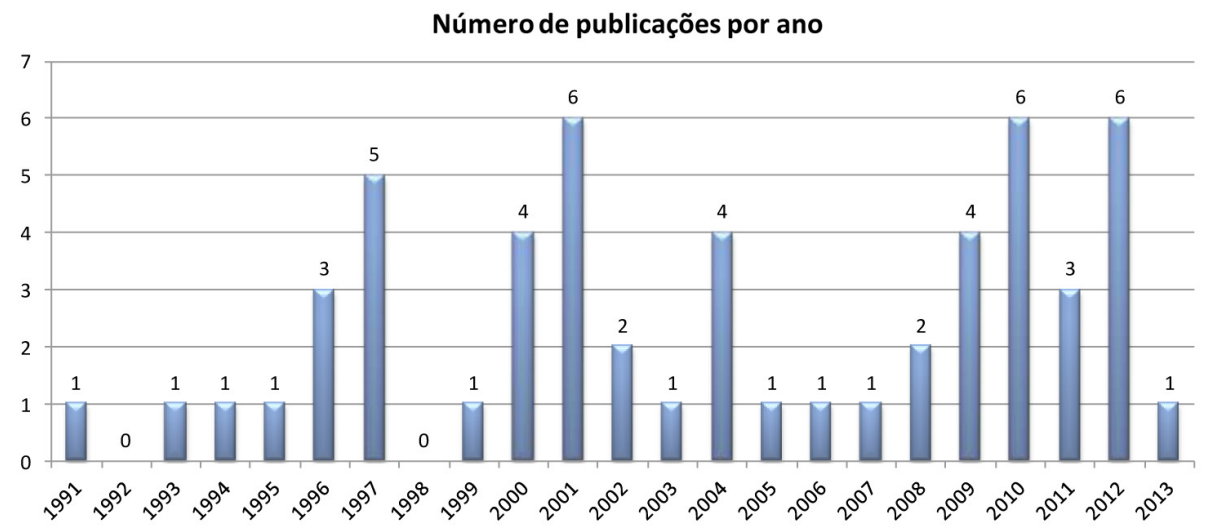

Figura 3. Número de publicações por ano contendo relações entre MC e Inovação. Fonte: Elaborado pelo autor.

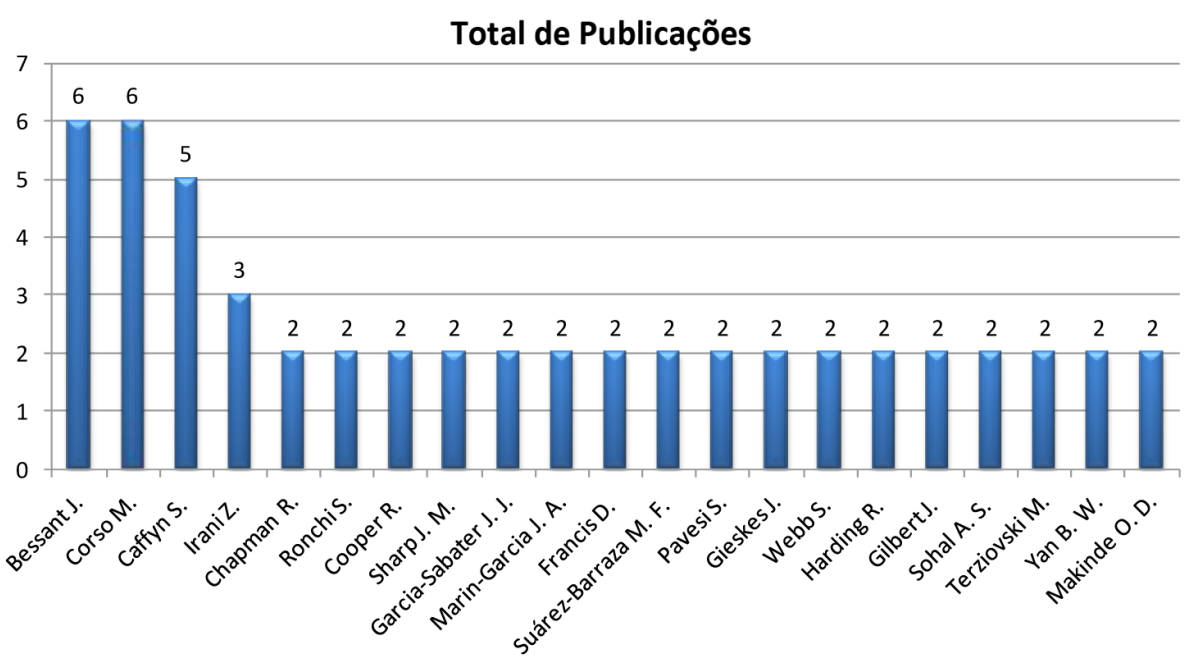

Figura 4. Número de publicações por autor. Fonte: Elaborado pelo autor.

Fator de Impacto e Número de Artigos

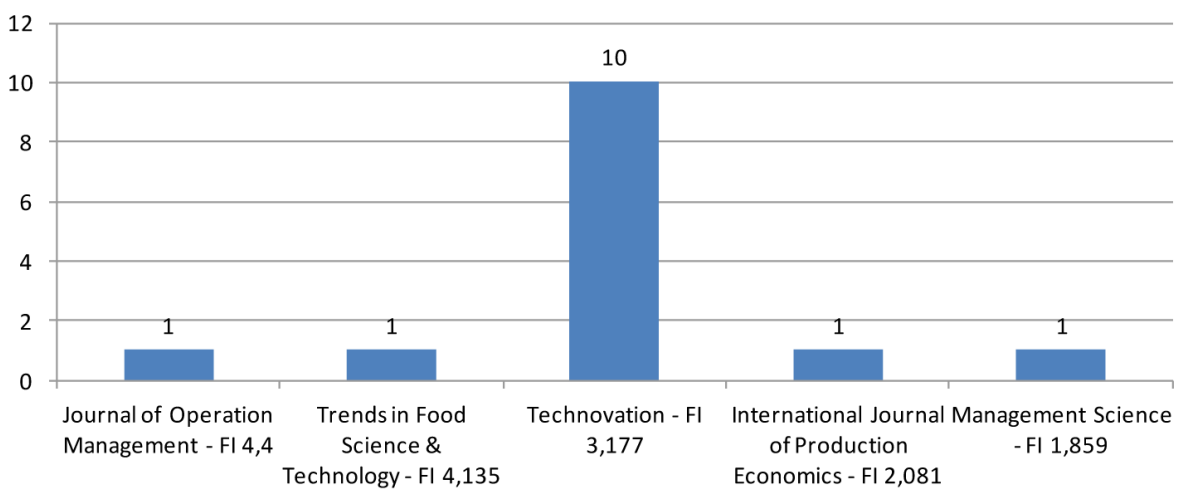

Figura 5. Número de publicações para as 5 revistas com maior fator de impacto. Fonte: Elaborado pelo autor. 


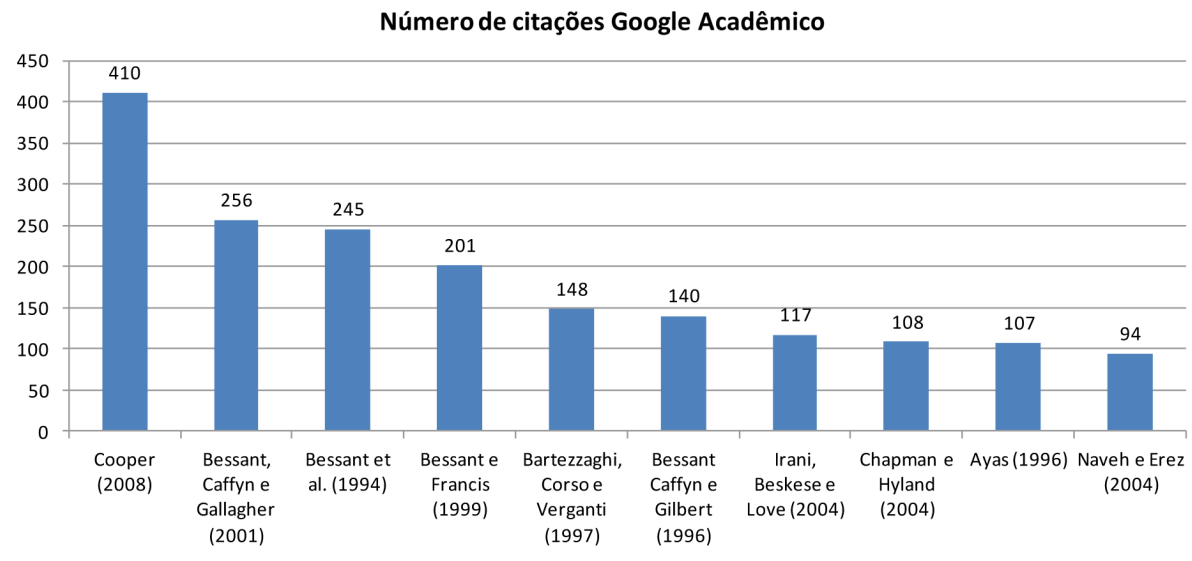

Figura 6. Publicações mais citadas (Google acadêmico). Fonte: Elaborado pelo autor.

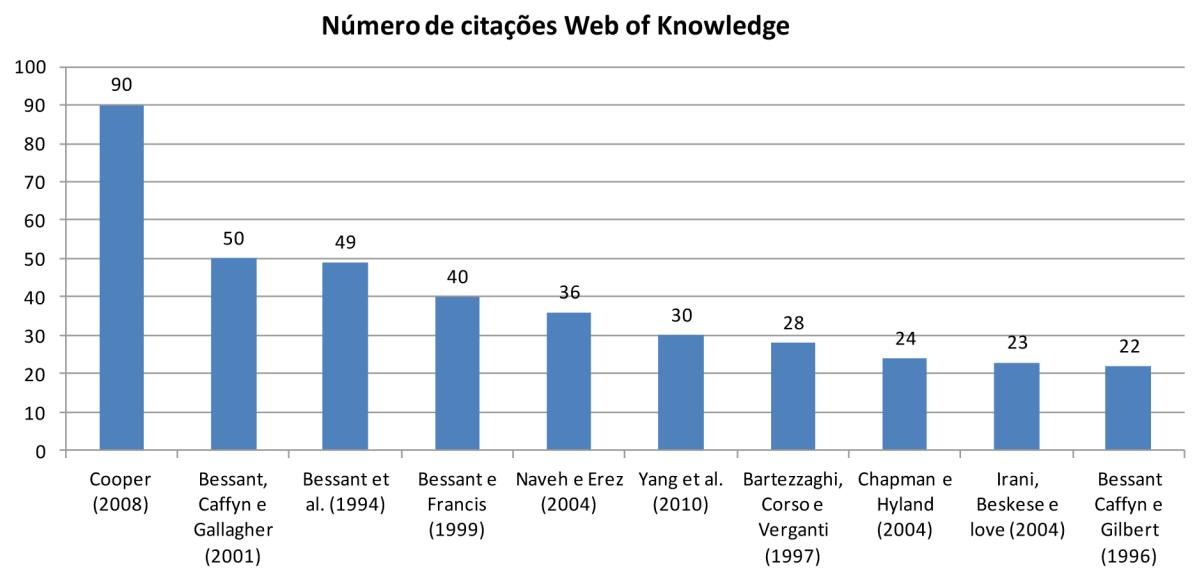

Figura 7. Publicações mais citadas (ISI Web of knowledge). Fonte: Elaborado pelo autor.

Tabela 2. Principais fontes de publicação.

\begin{tabular}{lcr}
\hline \multicolumn{1}{c}{ Fonte } & $\begin{array}{c}\text { Número de } \\
\text { Publicações }\end{array}$ & Porcentagem\% \\
\hline Technovation & 10 & 18,52 \\
International Journal of Technology Management & 4 & 7,41 \\
International Journal of Operations \& Production Management & 3 & 5,56 \\
IEEE International Conference on Management of Innovation and Technology & 3 & 5,56 \\
International Conference on Engineering, Technology and Innovation & 3 & 5,56 \\
African Journal of Business Management & 2 & 3,70 \\
The TQM magazine & 2 & 3,70 \\
Outros & 27 & 50,00 \\
\hline
\end{tabular}

Fonte: Elaborado pelo autor.

dezesseis tipos. As relações identificadas envolvem diferentes aspectos das organizações, por esse motivo foram separadas em três grandes categorias: apoio à inovação tecnológica (Quadro 3), cultura de inovação (Quadro 4) e aspectos colaborativos (Quadro 5). Algumas relações podem se encaixar em mais de uma categoria, porém escolheu-se aquela que mais refletia a relação analisada.

Nos Quadros 3, 4 e 5, para cada categoria, é apresentada a relação, a quantidade e a porcentagem de publicações, dentre as 54 analisadas, em que a relação está presente e os respectivos autores que as citam. No Quadro 3, há ainda uma divisão das relações com foco no escopo de aplicação: utilização de elementos da MC pela área de Inovação, geração de necessidades de projetos ou ações de MC alinhadas a projetos de Inovação e MC como geradora de inputs para a Inovação.

A atividade de identificação das relações apresentadas nas publicações teve algumas dificuldades, já que 
Quadro 3. Relações envolvendo apoio à inovação tecnológica.

\begin{tabular}{|c|c|c|c|}
\hline Relação identificada & $\begin{array}{l}\text { Quantidade } \\
\text { de } \\
\text { publicações }\end{array}$ & $\%$ & Autores \\
\hline \multicolumn{4}{|c|}{ Escopo: Utilização de elementos da MC pela área de Inovação } \\
\hline $\begin{array}{l}\text { Utilização de práticas } \\
\text { e ferramentas de } \\
\text { MC na gestão de } \\
\text { processo e projetos } \\
\text { da área de Pesquisa \& } \\
\text { Desenvolvimento \& } \\
\text { Inovação, incluindo o } \\
\text { PDP }\end{array}$ & 22 & 41 & $\begin{array}{l}\text { Kocaoglu et al. (1991); Bessant et al. (1994); Caffyn (1997); } \\
\text { Regan \& Kleiner (1997); Bessant \& Francis (1999); Berg et al. } \\
\text { (2001); Cole (2001); Sohal et al. (2003); Nilsson-Witell et al. } \\
\text { (2005); Middel et al. (2007); Cooper (2008); Prajogo \& Hong } \\
\text { (2008); Cooper (2009); Sun et al. (2009); Yan \& Makinde (2009); } \\
\text { Fàbregas-Fernández et al. (2010); Sandoval-Arzaga \& Suárez- } \\
\text { Barraza (2010); Kowang \& Rasli (2011); Yan \& Makinde (2011); } \\
\text { Rossi et al. (2012); Garcia-Sabater et al. (2012); Sopelana et al. } \\
(2012)\end{array}$ \\
\hline \multicolumn{4}{|c|}{ Escopo: Geração de necessidades de projetos ou ações de MC alinhadas a projetos de Inovação } \\
\hline $\begin{array}{l}\text { Geração de projetos } \\
\text { ou ações de melhoria } \\
\text { do processo alinhadas } \\
\text { à inovação de produtos }\end{array}$ & 10 & 19 & $\begin{array}{l}\text { Bartezzaghi et al. (1997); Earle (1997); Regan \& Kleiner (1997); } \\
\text { Corso \& Pavesi (2000); Boer et al. (2001); Chapman et al. (2001); } \\
\text { Chapman \& Hyland (2004); Nilsson-Witell et al. (2005); O’Brien } \\
\text { \& O’Reilly (2010); Peças et al. (2012) }\end{array}$ \\
\hline $\begin{array}{l}\text { Geração de projetos } \\
\text { ou ações de melhoria } \\
\text { do produto alinhadas à } \\
\text { inovação de produtos }\end{array}$ & 8 & 15 & $\begin{array}{l}\text { Bartezzaghi et al. (1997); Regan \& Kleiner (1997); Corso \& Pavesi } \\
\text { (2000); Boer et al. (2001); Chapman et al. (2001); Sohal et al. } \\
\text { (2003); Chapman \& Hyland (2004); Nilsson-Witell et al. (2005) }\end{array}$ \\
\hline \multicolumn{4}{|c|}{ Escopo: MC como geradora de inputs para a Inovação } \\
\hline $\begin{array}{l}\text { Auxílio na geração de } \\
\text { inovação de produtos } \\
\text { por meio da melhoria } \\
\text { contínua de produtos }\end{array}$ & 19 & 35 & $\begin{array}{l}\text { Bartezzaghi et al. (1997); Earle (1997); Bessant \& Francis } \\
\text { (1999); Corso \& Pavesi (2000); Eklund (2000); Lee et al. (2000); } \\
\text { Bessant et al. (2001); Nijhof et al. (2002); Sohal et al. (2003); } \\
\text { Naveh \& Erez (2004); Prajogo \& Hong (2008); Sun et al. (2009); } \\
\text { Yan \& Makinde (2009); Marin-Garcia et al. (2010); McAdam et al. } \\
\text { (2010); Sandoval-Arzaga \& Suárez-Barraza (2010); Yang et al. } \\
\text { (2010); Yan \& Makinde (2011); Martini et al. (2013) }\end{array}$ \\
\hline $\begin{array}{l}\text { Auxílio na geração de } \\
\text { inovação de processo } \\
\text { por meio da melhoria } \\
\text { contínua do processo }\end{array}$ & 13 & 24 & $\begin{array}{l}\text { Bartezzaghi et al. (1997); Bessant \& Francis (1999); Corso \& } \\
\text { Pavesi (2000); Lee et al. (2000); Bessant et al. (2001); Nijhof et al. } \\
\text { (2002); Naveh \& Erez (2004); Marin-Garcia, et al. (2010); } \\
\text { McAdam et al. (2010); O’Brien \& O’Reilly (2010); Yang et al. } \\
\text { (2010); Suárez-Barraza \& Smith (2014); Martini et al. (2013) }\end{array}$ \\
\hline
\end{tabular}

Fonte: Elaborado pelo autor.

muitas vezes a relação não está explícita, diferentes autores utilizam termos diferentes para designar conteúdos sobre MC e Inovação e a compreensão da relação acaba dependendo da interpretação do texto. Foram escolhidas para detalhamento as práticas das relações da categoria de apoio à inovação tecnológica (Quadro 3), já que essas relações apresentaram maior número de citações e, em alguns artigos, foi possível identificar aspectos mais objetivos e explícitos de interação e colaboração entre pessoas e projetos de $\mathrm{MC}$ e de inovação tecnológica. O mesmo não ocorreu com as relações das outras duas categorias, no caso da cultura de inovação, as práticas não foram apresentadas de maneira perceptível e, no caso dos aspectos colaborativos, foram poucos os artigos que abordaram essas relações.

As relações estudadas, da categoria de apoio à inovação tecnológica, foram agrupadas em escopos similares, já apresentados no Quadro 3, e compilados no Quadro 6. O primeiro escopo envolve o uso de elementos da MC, como práticas, ferramentas e métodos pela área de Inovação, isto pode ocorrer para a gestão de processos e projetos de Pesquisa \& Desenvolvimento \& Inovação (P\&D\&I) de produtos e de processos, envolvendo, principalmente a melhoria do Processo de Desenvolvimento de Produtos (PDP) (relação 1). No caso desta relação, os elementos da MC aparecem como possibilidade de melhorar o próprio processo de geração de inovações, ou seja, as etapas e as atividades envolvidas.

$\mathrm{O}$ segundo escopo envolve o alinhamento dos projetos e ações de $\mathrm{MC}$ aos projetos de Inovação, tanto a inovação de produtos que gera melhoria de outros produtos em linha (relação 2) quanto a inovação de produtos que solicita projetos de melhoria de processos (relação 3), para aumento do desempenho.

O terceiro escopo está relacionado com a MC como geradora de inputs para a Inovação: a MC 
Quadro 4. Relações envolvendo aspectos de formação da cultura de inovação com apoio da cultura de MC.

\begin{tabular}{|c|c|c|c|}
\hline Relação identificada & $\begin{array}{l}\text { Quantidade } \\
\text { de } \\
\text { publicações }\end{array}$ & $\%$ & Autores \\
\hline $\begin{array}{l}\text { MC como elemento } \\
\text { de aprendizagem, } \\
\text { experimentação e } \\
\text { caminho para Inovação }\end{array}$ & 14 & 26 & $\begin{array}{l}\text { Ayas (1996); Bartezzaghi et al. (1997); Corso \& Pavesi (2000); } \\
\text { Lee et al. (2000); Terziovski \& Sohal (2000); Bessant et al. (2001); } \\
\text { Boer et al. (2001); Cole (2001); Chapman \& Hyland (2004); } \\
\text { McAdam et al. (2010); Kowang \& Rasli (2011); Martowidjojo \& } \\
\text { Alamsjah (2011); Garcia-Sabater et al. (2012); Martini et al. (2013) }\end{array}$ \\
\hline $\begin{array}{l}\text { Cultura de MC como } \\
\text { auxílio na geração da } \\
\text { cultura de inovação } \\
\text { e de inovações } \\
\text { subsequentes }\end{array}$ & 13 & 24 & $\begin{array}{l}\text { Bessant et al. (1996); Irani \& Sharp (1997); Irani et al. (1997); } \\
\text { Corso \& Pavesi (2000); Lee et al. (2000); Bessant et al. (2001); } \\
\text { Nijhof et al. (2002); Irani et al. (2004); Naveh \& Erez (2004); } \\
\text { Fàbregas-Fernández et al. (2010); Marin-Garcia et al. (2010); } \\
\text { O’Brien \& O’Reilly (2010); Yan \& Makinde (2011) }\end{array}$ \\
\hline $\begin{array}{l}\text { MC como base cultural } \\
\text { de formação para a } \\
\text { inovação de produtos } \\
\text { (trabalho em equipe, } \\
\text { participação, geração } \\
\text { de ideias, entre outros) }\end{array}$ & 9 & 17 & $\begin{array}{l}\text { Bessant et al. (1996); Bessant et al. (2001); Nijhof et al. (2002); } \\
\text { Naveh \& Erez (2004); Nilsson-Witell et al. (2005); Sun et al. } \\
\text { (2009); McAdam et al. (2010); Kowang \& Rasli (2011); } \\
\text { Martini et al. (2013) }\end{array}$ \\
\hline $\begin{array}{l}\text { MC como base cultural } \\
\text { de formação para a } \\
\text { inovação de processos } \\
\text { (trabalho em equipe, } \\
\text { participação, geração } \\
\text { de ideias, entre outros) }\end{array}$ & 8 & 15 & $\begin{array}{l}\text { Bessant et al. (1996); Bessant et al. (2001); Nijhof et al. (2002); } \\
\text { Naveh \& Erez (2004); Nilsson-Witell et al. (2005); Anand et al. } \\
\text { (2009); McAdam et al. (2010); Martini et al. (2013) }\end{array}$ \\
\hline $\begin{array}{l}\text { MC como forma de } \\
\text { possibilitar geração } \\
\text { e transmissão de } \\
\text { conhecimento dentro } \\
\text { e entre projetos de } \\
\text { Inovação }\end{array}$ & 7 & 13 & $\begin{array}{l}\text { Ayas (1996); Bartezzaghi et al. (1997); Corso \& Pavesi (2000); } \\
\text { Boer et al. (2001); Chapman et al. (2001); Chapman \& Hyland } \\
\text { (2004); Martini et al. (2013) }\end{array}$ \\
\hline
\end{tabular}

Fonte: Elaborado pelo autor.

como apoiadora e geradora de ideias, tanto a MC de processos para a Inovação de processos (relação 4) quanto a $\mathrm{MC}$ de produtos gerando ideias para a Inovação de produtos (relação 5).

As relações 2, 3 e 5 englobam os projetos de desenvolvimento de novos produtos, enquanto a relação 1 engloba a melhoria do próprio processo de desenvolvimento de produto (por exemplo a sua gestão e desempenho).

A Figura 8 apresenta uma estrutura conceitual com as relações selecionadas, os números estão associados a cada uma das relações citadas no Quadro 6. Do lado esquerdo da figura estão representadas as atividades de MC (de produtos e de processos) e as ferramentas, técnicas e práticas associadas à MC. Do lado direito estão as atividades da área de Inovação (de produtos e de processos). A Figura apresenta conexões entre esses elementos.

Pode-se perceber que existem relações que são originadas na $\mathrm{MC}$, tanto em projetos de $\mathrm{MC}$ como na própria filosofia da $\mathrm{MC}$ que envolve ferramentas, técnicas e práticas. As relações fazem com que ideias ou mesmo as ferramentas e práticas originadas na MC migrem para projetos ou áreas da Inovação, estas são as relações 1, 4 e 5 . O fluxo inverso também pode ocorrer, ou seja, solicitações geradas por projetos da área de Inovação para projetos e ações de $\mathrm{MC}$, que são as relações 2 e 3.

Das dezesseis relações identificadas, a maioria apresenta uma relação, de causa e efeito, em que a origem é na MC e gera impactos na Inovação, tanto de produtos como de processos. As relações que envolvem aspectos culturais realçam o apoio da cultura da MC para formação de uma cultura de Inovação. As relações de colaboração envolvem aspectos de contribuição, ou auxílio mútuo para a estratégia competitiva da organização, mas ainda são relações pouco exploradas. Ainda é pouco frequente na literatura a citação de relações que envolvam a área ou projetos de Inovação solicitando ações ou contribuições da MC.

Para as relações de apoio à inovação tecnológica, foram identificadas as práticas de suporte, que podem ser vistas no Quadro 7. Alguns benefícios podem ser apontados em decorrência do uso das relações e das práticas. 
Para a utilização de práticas e ferramentas da MC pela área de Inovação, é necessário que haja interação, e troca de experiências, entre as pessoas ligadas à $\mathrm{MC}$ e as pessoas que executam e gerenciam os projetos de P\&D\&I. As práticas associadas evidenciam a necessidade de migração de ferramentas, técnicas e práticas da MC para o PDP e outros processos de pesquisa e desenvolvimento. A primeira relação apresenta a MC como forma de melhorar os processos envolvidos na área de $\mathrm{P} \& D \& I$, principalmente o desenvolvimento de novos produtos.

O objetivo da melhoria do próprio PDP é buscar a sua eficiência e eficácia, sem retrabalho e desperdício de tempo em atividades que não agregam valor ao processo (Yan \& Makinde, 2009; Kowang \& Rasli, 2011). Para a melhoria do PDP podem ser empregadas ferramentas da abordagem Lean como o Mapa de Fluxo de Valor e eliminação de desperdícios (Cooper, 2009; Sopelana et al. 2012), porém o uso destas ferramentas e práticas para a melhoria do PDP ainda permanece restrito (Rossi et al., 2012). As empresas procuram estratégias eficazes para melhorar a gestão do seu PDP, a fim de serem competitivas, pois a eficácia desse processo pode ser tão importante para a competitividade quanto os próprios produtos inovadores (Yan \& Makinde, 2011).

Quadro 5. Relações envolvendo aspectos colaborativos entre MC e Inovação.

\begin{tabular}{|l|c|c|l|}
\hline \multicolumn{1}{|c|}{ Relação identificada } & $\begin{array}{c}\text { Quantidade } \\
\text { de } \\
\text { publicações }\end{array}$ & $\%$ & Autores \\
\hline $\begin{array}{l}\text { MC e a Inovação devem estar } \\
\text { intimamente relacionadas e os } \\
\text { projetos estabelecem um papel } \\
\text { de colaboração }\end{array}$ & 6 & 11 & $\begin{array}{l}\text { Boer et al. (2001); Cole (2001); Chapman \& Hyland } \\
\text { (2004); Jager et al. (2004); Marin-Garcia et al. (2010); } \\
\text { Martini et al. (2013) }\end{array}$ \\
\hline $\begin{array}{l}\text { Complementaridade entre } \\
\text { projetos de MC e de Inovação }\end{array}$ & 2 & 4 & Bessant et al. (1994); Jager et al. (2004) \\
\hline $\begin{array}{l}\text { Integração entre ferramentas de } \\
\text { MC e de Desenvolvimento de } \\
\text { Produto }\end{array}$ & 1 & 2 & He et al. (2006) \\
\hline $\begin{array}{l}\text { Colaboração entre projetos } \\
\text { de MC e de Inovação } \\
\text { organizacional }\end{array}$ & 1 & 2 & MacDonald (1995) \\
\hline $\begin{array}{l}\text { Melhoria de processos } \\
\text { participando e contribuindo } \\
\text { para o melhor desempenho de } \\
\text { projetos de inovação }\end{array}$ & 1 & Anand et al. (2009) \\
\hline $\begin{array}{l}\text { Balanceamento de forma } \\
\text { colaborativa entre projetos de } \\
\text { MC e de Inovação }\end{array}$ & 2 & Oliveira et al. (2012) \\
\hline
\end{tabular}

Fonte: Elaborado pelo autor.

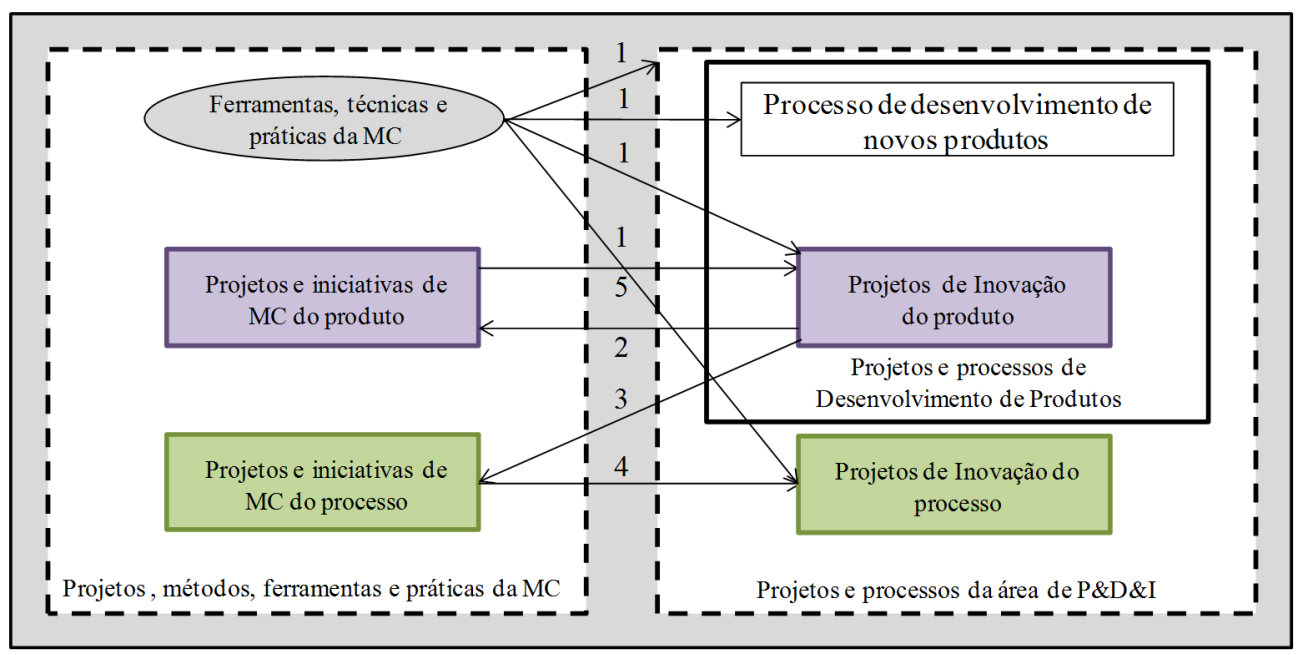

Figura 8. Estrutura conceitual das principais relações entre MC e Inovação. Fonte: Elaborado pelo autor. 
Outros autores salientam a utilização de ferramentas e práticas de $\mathrm{MC}$ nos processos da área de $\mathrm{P} \& D \& \mathrm{I}$ (Cole, 2001; Fàbregas-Fernández et al., 2010; Prajogo \& Hong, 2008; Garcia-Sabáter et al., 2012), obtendo resultados tais como menos erros na documentação de projetos, melhor avaliação da satisfação do cliente, bem como a implementação eficaz dos planos e objetivos (Fàbregas-Fernández et al., 2010) e mesmo aumento na inovação de produtos (Middel et al., 2007).

As demais relações envolvem, além de interação, a colaboração entre os projetos e ações da $\mathrm{MC}$ e da Inovação, necessitando-se que a organização se estruture para incentivar as práticas relacionadas. Muitas das práticas envolvem alinhamento de objetivos (principalmente as práticas para o escopo de geração de necessidades de projetos ou ações de MC alinhadas a projetos de Inovação) e troca de informações e de ideias (principalmente as práticas para o escopo da MC como geradora de inputs para a Inovação), uma vez que sem interação e colaboração entre as pessoas envolvidas não é possível que as relações sejam desenvolvidas. Os benefícios que podem ser gerados, mostrados no Quadro 7, requerem maior coordenação e alinhamento entre projetos e pessoas de diferentes áreas, gerando maior difusão de informações e melhores índices de desempenho de produtos e processos.

As práticas, tanto de geração de necessidades de projetos ou ações de $\mathrm{MC}$ alinhadas a projetos de Inovação quanto de MC como geradora de inputs para a Inovação, estão relacionadas com o continuum proposto na Figura 1, que mostra que o foco da MC e da Inovação são similares, diferindo no grau da mudança. Dessa forma, pode haver

Quadro 6. Relações de apoio à inovação tecnológica e respectivos escopos.

\begin{tabular}{|c|c|c|}
\hline Escopo & No. & Relações \\
\hline $\begin{array}{l}\text { Utilização de elementos da } \\
\text { MC pela área de Inovação }\end{array}$ & 1 & $\begin{array}{l}\text { Utilização de práticas e ferramentas de MC na gestão de processo e projetos da } \\
\text { área de Pesquisa \& Desenvolvimento \& Inovação, incluindo o PDP }\end{array}$ \\
\hline \multirow{2}{*}{$\begin{array}{l}\text { Geração de necessidades } \\
\text { de projetos ou ações de } \\
\text { MC alinhadas a projetos } \\
\text { de Inovação }\end{array}$} & 2 & $\begin{array}{l}\text { Geração de projetos ou ações de melhoria do produto alinhadas à inovação de } \\
\text { produtos }\end{array}$ \\
\hline & 3 & $\begin{array}{l}\text { Geração de projetos ou ações de melhoria do processo alinhadas à inovação de } \\
\text { produtos }\end{array}$ \\
\hline \multirow{2}{*}{$\begin{array}{l}\text { MC como geradora de } \\
\text { inputs para a Inovação }\end{array}$} & 4 & $\begin{array}{l}\text { Auxílio na geração de inovação de processo por meio de melhorias de } \\
\text { processos }\end{array}$ \\
\hline & 5 & Auxílio na geração de inovação de produtos por meio da melhoria de produtos \\
\hline
\end{tabular}

Fonte: Elaborado pelo autor.

Quadro 7. Relações, práticas e implicações e benefícios gerenciais.

\begin{tabular}{|c|c|c|c|}
\hline Relações & $\begin{array}{c}\text { Definição, benefícios e implicações da } \\
\text { relação }\end{array}$ & Práticas & Autores das práticas \\
\hline \multirow{4}{*}{$\begin{array}{l}\text { 1- Utilização } \\
\text { de práticas e } \\
\text { ferramentas de } \\
\text { MC na gestão } \\
\text { de processo e } \\
\text { projetos da área } \\
\text { de Pesquisa \& } \\
\text { Desenvolvimento } \\
\text { \& Inovação, } \\
\text { incluindo o PDP }\end{array}$} & \multirow{4}{*}{$\begin{array}{l}\text { Definição: } \\
\text { Melhoria no próprio PDP e auxílio no } \\
\text { desenvolvimento das etapas de projetos } \\
\text { de Pesquisa \& Desenvolvimento \& } \\
\text { Inovação por meio da utilização de } \\
\text { ferramentas e práticas da MC } \\
\text { Benefícios: } \\
\text { Maior eficiência, otimização, redução de } \\
\text { tempo de desenvolvimento e redução de } \\
\text { custos e de desperdícios. } \\
\text { Aplicação das ferramentas, práticas } \\
\text { e técnicas da MC para auxílio } \\
\text { no desenvolvimento de projetos, } \\
\text { possibilitando pontos de vistas } \\
\text { diferenciados pelo uso das ferramentas. } \\
\text { Implicações: } \\
\text { A organização deve possibilitar a } \\
\text { interação entre pessoas das áreas de } \\
\text { MC e de Pesquisa e Desenvolvimento, } \\
\text { incluindo pessoas do PDP, para fomentar } \\
\text { conhecimento e uso das práticas e } \\
\text { ferramentas. }\end{array}$} & $\begin{array}{l}\text { Utilizar ferramentas e } \\
\text { técnicas da MC, como o } \\
\text { Mapa de Fluxo de Valor, } \\
\text { para a identificação } \\
\text { e eliminação de } \\
\text { desperdícios. }\end{array}$ & $\begin{array}{l}\text { Kocaoglu et al. (1991); } \\
\text { Caffyn (1997); Cooper } \\
\text { (2008), Cooper (2009); } \\
\text { Sun et al. (2009); Yan } \\
\text { \& Makinde (2009); } \\
\text { Rossi et al. (2012); } \\
\text { Sopelana et al. (2012) }\end{array}$ \\
\hline & & $\begin{array}{l}\text { Analisar as causas de } \\
\text { problemas no PDP. }\end{array}$ & $\begin{array}{l}\text { Cooper (2008), Cooper } \\
(2009)\end{array}$ \\
\hline & & $\begin{array}{l}\text { Não culpar equipe do } \\
\text { PDP, quando problemas } \\
\text { ocorrem. }\end{array}$ & $\begin{array}{l}\text { Cooper (2008), Cooper } \\
(2009)\end{array}$ \\
\hline & & $\begin{array}{l}\text { Aplicar métricas de } \\
\text { desempenho no PDP } \\
\text { visando identificar } \\
\text { possibilidades de } \\
\text { melhorias e aplicá-las. }\end{array}$ & $\begin{array}{l}\text { Regan \& Kleiner (1997); } \\
\text { Cooper (2008), Cooper } \\
\text { (2009); Rossi et al. } \\
\text { (2012); Sopelana et al. } \\
(2012)\end{array}$ \\
\hline
\end{tabular}


Quadro 7. Continuação...

\begin{tabular}{|c|c|c|c|}
\hline Relações & $\begin{array}{c}\text { Definição, benefícios e implicações da } \\
\text { relação }\end{array}$ & Práticas & Autores das práticas \\
\hline \multirow{3}{*}{$\begin{array}{l}\text { 2- Geração de } \\
\text { projetos ou ações } \\
\text { de melhoria do } \\
\text { produto alinhadas } \\
\text { à inovação de } \\
\text { produtos }\end{array}$} & \multirow{3}{*}{$\begin{array}{l}\text { Definição: } \\
\text { Alinhar os projetos de melhoria } \\
\text { de produtos existentes com o } \\
\text { desenvolvimento de novos produtos. } \\
\text { Benefícios: } \\
\text { Minimização de esforços e } \\
\text { desperdícios com ações conjuntas entre } \\
\text { desenvolvimento de novos produtos e } \\
\text { melhoria de produtos existentes, gerando } \\
\text { melhor desempenho dos produtos para os } \\
\text { clientes. } \\
\text { Implicações: } \\
\text { A organização deve possibilitar a } \\
\text { interação e colaboração entre projetos e } \\
\text { pessoas das áreas de MC de produtos e da } \\
\text { Inovação de produtos. }\end{array}$} & $\begin{array}{l}\text { Usar os objetivos da área } \\
\text { de desenvolvimento para } \\
\text { focar as atividades de } \\
\text { melhoria de produtos. }\end{array}$ & $\begin{array}{l}\text { Corso \& Pavesi (2000); } \\
\text { Boer et al. (2001); } \\
\text { Chapman et al. (2001); } \\
\text { Corso (2002); Chapman } \\
\text { \& Hyland (2004) }\end{array}$ \\
\hline & & $\begin{array}{l}\text { Estimular melhoria } \\
\text { das características dos } \\
\text { produtos existentes e } \\
\text { usar parte do tempo e } \\
\text { recursos disponíveis para } \\
\text { experimentar soluções. }\end{array}$ & $\begin{array}{l}\text { Regan \& Kleiner (1997); } \\
\text { Bessant \& Francis } \\
\text { (1999); Corso \& Pavesi } \\
\text { (2000); Boer et al. } \\
\text { (2001); Bessant et al. } \\
\text { (2001); Chapman et al. } \\
\text { (2001); Corso (2002); } \\
\text { Chapman \& Hyland } \\
\text { (2004); Jager et al. } \\
\text { (2004); Garcia- } \\
\text { Sabáter et al. (2012) }\end{array}$ \\
\hline & & $\begin{array}{l}\text { Incorporar conhecimento } \\
\text { da inovação de produtos } \\
\text { em relatórios, banco } \\
\text { de dados, entre outros, } \\
\text { para posterior acesso e } \\
\text { melhorias de produto. }\end{array}$ & $\begin{array}{l}\text { Bessant \& Francis } \\
\text { (1999); Corso \& Pavesi } \\
\text { (2000); Bessant et al. } \\
(2001) \text {; Boer et al. } \\
(2001) ; \text { Chapman et al. } \\
\text { (2001); Corso (2002); } \\
\text { Chapman \& Hyland } \\
\text { (2004); Jager et al. } \\
\text { (2004); Kowang \& } \\
\text { Rasli (2011); Garcia- } \\
\text { Sabáter et al. (2012) }\end{array}$ \\
\hline \multirow{3}{*}{$\begin{array}{l}\text { 3- Geração de } \\
\text { projetos ou ações } \\
\text { de melhoria } \\
\text { do processo } \\
\text { alinhadas à } \\
\text { inovação de } \\
\text { produtos }\end{array}$} & \multirow{3}{*}{$\begin{array}{l}\text { Definição: } \\
\text { Alinhar os projetos de MC de processos } \\
\text { com desenvolvimento de novos produtos. } \\
\text { Benefícios: } \\
\text { União de esforços (área de MC e área de } \\
\text { Inovação) para a melhoria do desempenho } \\
\text { do processo e do novo produto, podendo } \\
\text { utilizar o desenvolvimento do novo } \\
\text { produto para otimizar processos para os } \\
\text { produtos atuais que utilizam as mesmas } \\
\text { operações produtivas. } \\
\text { Implicações: } \\
\text { A organização deve possibilitar a } \\
\text { interação e colaboração entre projetos e } \\
\text { pessoas das áreas de MC de processos e } \\
\text { da Inovação de produtos. }\end{array}$} & $\begin{array}{l}\text { Usar os objetivos da área } \\
\text { de desenvolvimento para } \\
\text { focar as atividades de } \\
\text { melhoria de processo. }\end{array}$ & $\begin{array}{l}\text { Corso \& Pavesi (2000); } \\
\text { Boer et al. (2001); } \\
\text { Chapman et al. (2001); } \\
\text { Corso (2002); Chapman } \\
\text { \& Hyland (2004) }\end{array}$ \\
\hline & & $\begin{array}{l}\text { Estimular a melhoria } \\
\text { do processo de } \\
\text { fabricação durante o } \\
\text { desenvolvimento do } \\
\text { produto. }\end{array}$ & Regan \& Kleiner (1997) \\
\hline & & $\begin{array}{l}\text { Incorporar conhecimento } \\
\text { do desenvolvimento de } \\
\text { produtos em relatórios, } \\
\text { banco de dados, normas } \\
\text { de processo entre outros, } \\
\text { para posterior acesso e } \\
\text { melhorias de processos. }\end{array}$ & $\begin{array}{l}\text { Bessant \& Francis } \\
\text { (1999); Corso \& Pavesi } \\
(2000) \text {; Boer et al. } \\
(2001) ; \text { Bessant et al. } \\
(2001) ; \text { Chapman et al. } \\
\text { (2001); Corso (2002); } \\
\text { Chapman \& Hyland } \\
\text { (2004); Jager et al. } \\
\text { (2004); Kowang \& } \\
\text { Rasli (2011); Garcia- } \\
\text { Sabáter et al. (2012) } \\
\end{array}$ \\
\hline
\end{tabular}

Fonte: Elaborado pelo autor. 
Quadro 7. Continuação...

\begin{tabular}{|c|c|c|c|}
\hline Relações & $\begin{array}{c}\text { Definição, benefícios e implicações da } \\
\text { relação }\end{array}$ & Práticas & Autores das práticas \\
\hline \multirow{3}{*}{$\begin{array}{l}\text { 4- Auxílio } \\
\text { na geração } \\
\text { de inovação } \\
\text { de processo } \\
\text { por meio de } \\
\text { melhorias de } \\
\text { processos }\end{array}$} & \multirow{3}{*}{$\begin{array}{l}\text { Definição: } \\
\text { Estimular o uso do conhecimento e } \\
\text { ideias das pessoas da área de MC para } \\
\text { possibilitar também inovações mais } \\
\text { radicais de processos. Aproveitar as } \\
\text { ideias de melhoria geradas e incentivar o } \\
\text { uso em novos projetos, podendo utilizar } \\
\text { o conhecimento interno para novos } \\
\text { desenvolvimentos de processos. } \\
\text { Benefícios: } \\
\text { Melhor utilização de recursos, estímulo } \\
\text { à Inovação e motivação das pessoas } \\
\text { envolvidas em projetos de MC. } \\
\text { Implicações: } \\
\text { A organização deve possibilitar a } \\
\text { interação e colaboração entre projetos e } \\
\text { pessoas das áreas de MC de processos e } \\
\text { da Inovação de processos. }\end{array}$} & $\begin{array}{l}\text { Incentivar ideias de } \\
\text { inovação de processos } \\
\text { oriundas das pessoas } \\
\text { ligadas à MC de } \\
\text { processos. }\end{array}$ & $\begin{array}{l}\text { Regan \& Kleiner (1997); } \\
\text { Sohal et al. (2003); } \\
\text { O'Brien \& O'Reilly } \\
(2010)\end{array}$ \\
\hline & & $\begin{array}{l}\text { Gerar fluxo livre de } \\
\text { ideias e informações da } \\
\text { MC de processos para a } \\
\text { inovação de processos. }\end{array}$ & $\begin{array}{l}\text { Irani \& Sharp (1997); } \\
\text { Irani et al. (2004); } \\
\text { Jager et al. (2004) }\end{array}$ \\
\hline & & $\begin{array}{l}\text { Incentivar migração de } \\
\text { projetos de melhoria } \\
\text { contínua de processos } \\
\text { para projetos de } \\
\text { inovação de processos. }\end{array}$ & $\begin{array}{l}\text { Bessant et al. (2001); } \\
\text { Nilsson-Witell et al. } \\
\text { (2005); McAdam et al. } \\
\text { (2010); O'Brien \& } \\
\text { O'Reilly (2010) }\end{array}$ \\
\hline \multirow{3}{*}{$\begin{array}{l}\text { 5- Auxílio na } \\
\text { geração de } \\
\text { inovação de } \\
\text { produtos por } \\
\text { meio da melhoria } \\
\text { de produtos }\end{array}$} & \multirow{3}{*}{$\begin{array}{l}\text { Definição: } \\
\text { Estimular o uso do conhecimento das } \\
\text { pessoas da área de MC para possibilitar } \\
\text { também inovações mais radicais de } \\
\text { produtos. Aproveitar as ideias de melhoria } \\
\text { geradas e incentivar o uso em novos } \\
\text { projetos, podendo utilizar o conhecimento } \\
\text { interno para o desenvolvimento de novos } \\
\text { produtos. } \\
\text { Benefícios: } \\
\text { Melhor utilização de recursos, estímulo } \\
\text { à Inovação e motivação das pessoas } \\
\text { envolvidas em projetos de MC. } \\
\text { Implicações: } \\
\text { A organização deve possibilitar a } \\
\text { interação e colaboração entre projetos e } \\
\text { pessoas da MC de produtos e da Inovação } \\
\text { de produtos. }\end{array}$} & $\begin{array}{l}\text { Incentivar a geração de } \\
\text { ideias de inovação de } \\
\text { produtos das pessoas } \\
\text { ligadas à MC de produto. }\end{array}$ & $\begin{array}{l}\text { Regan \& Kleiner (1997); } \\
\text { Sohal, et al. (2003); } \\
\text { O'Brien \& O'Reilly } \\
(2010)\end{array}$ \\
\hline & & $\begin{array}{l}\text { Gerar fluxo livre de } \\
\text { ideias e informações da } \\
\text { MC de produtos para a } \\
\text { inovação de produtos. }\end{array}$ & $\begin{array}{l}\text { Irani \& Sharp (1997); } \\
\text { Irani et al. (2004); } \\
\text { Jager et al. (2004) }\end{array}$ \\
\hline & & $\begin{array}{l}\text { Incentivar a migração } \\
\text { de projetos de melhoria } \\
\text { contínua de produtos } \\
\text { para projetos de } \\
\text { inovação de produtos. }\end{array}$ & $\begin{array}{l}\text { Bessant et al. (2001); } \\
\text { Nilsson-Witell et al. } \\
\text { (2005); McAdam et al. } \\
\text { (2010); O’Brien \& } \\
\text { O'Reilly (2010) }\end{array}$ \\
\hline
\end{tabular}

Fonte: Elaborado pelo autor.

contribuições entre os projetos e ações de $\mathrm{MC}$ e de Inovação. Os projetos de melhoria de processos e de produtos podem auxiliar na geração de ideias e no comprometimento das pessoas em relação à geração de inovações menores e maiores (Bessant \& Francis, 1999; Bessant et al., 2001).

O conceito de que os esforços de projetos e ações de $\mathrm{MC}$ podem contribuir para inovações incrementais e radicais está principalmente baseado no modelo de maturidade da $\mathrm{MC}$ apresentado por Bessant et al. (2001), em que, em um de seus últimos níveis de evolução, o aprendizado em MC possibilita o envolvimento das pessoas no processo de inovação (Bessant et al., 1996, 2001; Caffyn, 1999; Bessant \& Francis, 1999). Bessant é o autor com maior número de trabalhos que relacionam os dois temas (MC e Inovação) e quatro dos artigos relacionados aos níveis de maturidade, em que ele contribuiu, estão entre os dez mais citados da RBS.

O conceito de inovação contínua do produto foi uma das principais bases de conhecimento para as relações em que projetos de MC geram inputs e estão alinhados a projetos de Inovação. Corso, um dos principais autores que aborda esse conceito, aparece como segundo autor com maior número de artigos que relacionam MC e Inovação. Dois artigos relacionados com a inovação contínua do produto (Bartezzaghi et al., 1997; Chapman \& Hyland, 2004) aparecem entre os dez mais citados.

O conceito da inovação contínua do produto relaciona a $\mathrm{MC}$, e suas variáveis de aprendizado e difusão do conhecimento, com o desenvolvimento de novos produtos, relacionando melhoria de processo e de produto com o processo de inovação (Corso \& 
Pavesi, 2000; Chapman et al., 2001; Corso, 2002; Chapman \& Hyland, 2004). A inovação contínua de produto salienta tanto as relações de geração de inovações decorrentes da MC quanto de alinhamentos entre MC e Inovação.

\section{Considerações finais e possibilidades para pesquisas futuras}

A RBS possibilitou a análise das principais referências bibliográficas sobre o tema de pesquisa e trouxe embasamento teórico para a criação de uma estrutura conceitual das relações selecionadas. Ainda por meio da RBS foi possível identificar práticas que sustentam essas relações e inferir sobre implicações e benefícios gerenciais gerados pelo uso das práticas.

Como pôde ser observado na análise dos resultados, as referências bibliográficas que continham o conhecimento sobre conexões entre MC e Inovação estavam distribuídas em mais de duas décadas. A RBS permitiu a reunião dessas informações e possibilitou, também, verificar a importância das fontes em que esses artigos foram publicados, comprovando a importância do tema pesquisado e possibilitando que futuros pesquisadores direcionem a fonte de suas pesquisas e futuras publicações.

A identificação das relações mostrou que o tema e a aplicação nas organizações são bastante amplos, e que as relações identificadas envolvem diferentes aspectos da gestão organizacional, como cultura, ferramentas e técnicas de gestão de projetos, colaboração entre equipes e projetos e interação entre pessoas de áreas distintas. A síntese das informações dos artigos e das relações identificadas foi uma atividade complexa, já que os autores definem de formas diversas essas conexões e os próprios conceitos sobre MC e Inovação podem diferir entre os autores, o que dificulta ainda mais a análise e evidencia a necessidade de estudos mais aprofundados.

Dentre as dezesseis relações identificadas, foi possível perceber que 5 delas apresentam a MC como impulsionadora da cultura de Inovação, da experimentação, da transmissão de conhecimento necessário para a gestão da inovação e como base cultural para impulsionar a participação de todos na empresa no processo de inovação. Esse conceito é relevante no cenário atual de inovação aberta com a participação de clientes, fornecedores e membros externos e internos da organização.

Também foi possível notar que há relações envolvendo aspectos colaborativos entre MC e Inovação, uma forma de balancear e mesmo focar conjuntamente ações e ferramentas, porém os trabalhos sobre esse tópico ainda são muito escassos e não é possível realizar afirmações sobre práticas e perspectivas de aplicação.
As relações envolvendo apoio à inovação tecnológica, foco principal do artigo, apresentam conexões mais diretas com o processo de inovação, seja propondo melhorias de processos organizacionais, como o PDP e outros processos da área de $\mathrm{P} \& \mathrm{D} \& \mathrm{I}$, ou relações ainda mais diretas, como o alinhamento e colaboração entre projetos e ações de MC e de Inovação. A relação envolvendo utilização de elementos da MC pela área de Inovação foi a mais citada nos artigos, mostrando a sua importância. As relações de geração de necessidades de projetos ou ações de MC alinhadas a projetos de Inovação, e de MC como geradora de inputs para a Inovação, estão mais associadas com a ideia do continuum proposto na Figura 1 e com as sobreposições de foco entre MC e Inovação. Estas relações apresentam possibilidades de colaboração entre pessoas, projetos e ações com foco na melhor utilização de recursos, buscando melhor desempenho de produtos e processos.

As relações envolvendo apoio à inovação tecnológica estão mais estruturadas em alguns artigos, outros apenas as citam de forma sucinta, mas foi possível elencar práticas e maneiras de impulsionar essas relações nas organizações.

Há muitas possibilidades de desenvolvimento de modelos teóricos e explicativos de relações entre a MC e a Inovação, envolvendo, por exemplo, aspectos culturais e suas implicações. Outra possibilidade é a mensuração da correlação entre o uso das relações e suas práticas e das relações e o desempenho, seja da Inovação e da qualidade de produtos e processos. A investigação sobre o uso de relações e práticas e dos resultados obtidos pode ser voltada a setores industriais específicos, pois há falta de trabalhos empíricos sobre o tema. O Quadro 8 apresenta algumas possibilidades de pesquisas futuras.

A construção desta primeira estrutura conceitual de relações e a identificação das práticas associadas é o ponto inicial para pesquisas futuras sobre os benefícios e dificuldades dessas relações, e sobre a verificação da utilização das práticas encontradas e de outras existentes nas organizações. Apenas cinco relações foram analisadas em maior profundidade; outros estudos podem focar outras relações encontradas na RBS. Pesquisas futuras podem ser direcionadas para estudar organizações de setores econômicos específicos, podendo-se considerar a possível influência, nas relações e práticas estudadas, de fatores contingenciais como a estratégia tecnológica utilizada pela organização e fatores externos, como o mercado e o ritmo de mudança das tecnologias envolvidas.

Acredita-se que esta pesquisa seja capaz de apresentar e sintetizar o que existe publicado sobre este tema, já que houve uma preocupação com os procedimentos de pesquisa utilizados, buscando-se a identificação do estado da arte sobre o tema e a redução de vieses, a utilização de quatro amplas bases de periódicos relevantes e a análise e seleção de estudos publicados nos últimos 25 anos (1989 a 2013). 
Quadro 8. Oportunidades de temas para realização de pesquisas futuras.

Criação de modelos de relações, práticas e resultados e mensuração da correlação entre o uso das práticas e relações e o desempenho da Inovação e da qualidade de produtos e processos

Criação e validação de modelos incluindo outras relações e práticas além das cinco investigadas neste artigo Proposição de modelos de relações entre MC e Inovação, práticas e resultados voltados para organizações de setores econômicos específicos

Análise das práticas que mais auxiliam na consolidação das relações e identificação de outras por meio de pesquisa de campo

Criação de modelo de maturidade para avaliação e evolução das relações entre MC e Inovação

Expansão da análise para outras conexões entre MC e Inovação, tais como melhorias e inovações de processos organizacionais

Fonte: Elaborado pelo autor.

\section{Referências}

Abernathy, W. J., \& Utterback, J. M. (1978). Patterns of innovation in industry. Technology Review, 80(7), 40-47.

Adler, P., Goldoftas, B., \& Levine, D. (1999). Flexibility versus efficiency? A case study of model changeovers in the Toyota production system. Organization Science, 10(1), 43-68. http://dx.doi.org/10.1287/orsc.10.1.43.

Anand, G., Ward, P. T., Tatikonda, M. V., \& Schilling, D. A. (2009). Dynamic capabilities through continuous improvement infrastructure. Journal of Operations Management, 27(6), 444-461. http://dx.doi.org/10.1016/j. jom.2009.02.002.

Andersson, R., Eriksson, H., \& Torstensson, H. (2006). Similarities and differences between TQM, six sigma and lean. The TQM Magazine, 18(3), 282-296. http:// dx.doi.org/10.1108/09544780610660004.

Ayas, K. (1996). Professional project management: a shift towards learning and a knowledge creating structure. International Journal of Project Management, 14(3), 131-136. http://dx.doi.org/10.1016/0263-7863(95)00080-1.

Bartezzaghi, E., Corso, M., \& Verganti, R. (1997). Int. J. Continuous improvement and inter-project learning in new product development. Technology Management, 14(1), 116-138.

Benner, M. J., \& Tushman, M. L. (2003). Exploitation, Exploration, and Process Management: The Productivity Dilemma Revisited. Academy of Management Review, 28, 238-256.

Berg, P., Pihlajamaa, J., Leinonen, M., \& Leivo, V. (2001). Assessment of Quality and Maturity Level of R\&D. In Proceedings of the PICMET '01 (pp. 168-182). Portland.

Bessant, J., \& Francis, D. (1999). Developing Strategic Continuous Improvement Capability. International Journal of Operations \& Production Management, 19(11), 11061119. http://dx.doi.org/10.1108/01443579910291032.

Bessant, J., Caffyn, S., \& Gallagher, M. (2001). An evolutionary model of continuous improvement behavior. Technovation, 21(2), 67-77. http://dx.doi.org/10.1016/ S0166-4972(00)00023-7.
Bessant, J., Caffyn, S., \& Gilbert, J. (1996). Learning to Manage Innovation. Technology Analysis and Strategic Management, 8(1), 59-70. http://dx.doi. org/10.1080/09537329608524233.

Bessant, J., Caffyn, S., Gilbert, J., Harding, R., \& Webb, S. (1994). Rediscovering continuous improvement. Technovation, 14(1), 17-29. http://dx.doi.org/10.1016/01664972(94)90067-1.

Bhuiyan, N., \& Baghel, A. (2005). An overview of continuous improvement: from the past to the present. Management Decision, 43(5), 761-771. http://dx.doi. org/10.1108/00251740510597761.

Bhuiyan, N., Baghel, A., \& Wilson, J. (2006). WILSON, J. A sustainable continuous improvement methodology at an aerospace company. International Journal of Productivity and Performance Management, 55(8), 671-687. http://dx.doi.org/10.1108/17410400610710206.

Biolchini, J., Mian, P. G., Natali, A. C. C., Conte, T. U., \& Travessos, G. H. (2007). Scientific research ontology to support systematic review in software engineering. Advanced Engineering Informatics, 21(2), 133-151. http://dx.doi.org/10.1016/j.aei.2006.11.006.

Boer, H., \& Gertsen, F. (2003). From continuous improvement to continuous innovation: a (retro)(per)spective. International Journal of Technology Management, 26(8), 805-827. http://dx.doi.org/10.1504/IJTM.2003.003391.

Boer, H., Caffyn, S., Corso, M., Coughlan, P., Gieskes, J., Magnusson, M., Pavesi, S., \& Ronchi, S. (2001). Knowledge and continuous innovation: the CIMA methodology. International Journal of Operations \& Production Management, 21(4), 490-504. http://dx.doi. org/10.1108/01443570110381390.

Brereton, P., Kitchenham, B. A., Budgen, D., Turner, M., \& Khalil, M. (2007). Lessons from applying the systematic literature review process within the software engineering domain. Journal of Systems and Software, 80(4), 571583. http://dx.doi.org/10.1016/j.jss.2006.07.009.

Caffyn, S. (1997). Extending continuous improvement to the new product development process. $R \& D$ Management, 27(3), 253-267. http://dx.doi.org/10.1111/1467-9310.00061.

Caffyn, S. (1999). Development of a continuous improvement selfassessment tool. International Journal of Operations 
\& Production Management, 19(11), 1138-1153. http:// dx.doi.org/10.1108/01443579910291050.

Caffyn, S., \& Bessant, J. (1996). A capability-based model for continuous improvement. In Proceedings of 3 th International Conference of the EUROMA. London.

Cambridge. Cambridge Advanced Learner's Dictionary. Recuperado em fevereiro de 2013, de http://dictionary. cambridge.org/

Chapman, R. L., \& Corso, M. (2005). From continuous improvement to collaborative innovation: the next challenge in supply chain management. Production Planning and Control, 16(4), 339-344. http://dx.doi. org/10.1080/09537280500063269.

Chapman, R., \& Hyland, P. (2004). Complexity and learning behaviors in product innovation. Technovation, 24(7), 553561. http://dx.doi.org/10.1016/S0166-4972(02)00121-9.

Chapman, R., O’Mara, C. E., Ronchi, S., \& Corso, M. (2001). Continuous product innovation: A comparison of key elements across different contingency sets. Measuring Business Excellence, 5(3), 16-23. http:// dx.doi.org/10.1108/EUM0000000005866.

Cole, R. E. (2001). From continuous improvement to continuous innovation. Quality Management Journal, 8(4), 7-21.

Cole, R. E., \& Matsumiya, T. (2008). When the pursuit of quality risks innovation. The TQM Journal, 20(2), 130142. http://dx.doi.org/10.1108/17542730810857363.

Cooper, R. (2008). Perspective: The Stage-Gates Idea-toLaunch Process - Update, What's New, and NexGen Systems. Journal of Product Innovation Management, 25(3), 213-232. http://dx.doi.org/10.1111/j.15405885.2008.00296.x.

Cooper, R. (2009). How companies are reinventing their Idea-to-launch methodologies. Technology Management, 52(2), 47-57.

Corso, M. (2002). From product development to Continuous Product Innovation: mapping the routes of corporate knowledge. International Journal of Technology Management, 23(4), 322. http://dx.doi.org/10.1504/ IJTM.2002.003013.

Corso, M., \& Gastaldi, L. (2011). Toward a relevant, reflective and rigorous methodology able to study continuous innovation at affordable resource-consumptions levels. In Proceedings of the 12 Continuous Innovation Network - CINET. Aarhus: Aarhus University. Recuperado em 18 de janeiro de 2012, de http://www.continuous-innovation. net/members-area/proceedings/2011/corso-gastaldicinet2011.pdf

Corso, M., \& Pavesi, S. (2000). How management can foster continuous product innovation. Integrated Manufacturing Systems, 11(3), 199-211. http://dx.doi. org/10.1108/09576060010320443.

Corso, M., \& Pellegrini, L. (2007). Continuous and discontinuous innovation: overcoming the innovator dilemma. Creativity and Innovation Management, 16(4), 333-347. http://dx.doi.org/10.1111/j.14678691.2007.00459.x.

Damanpour, F. (1987). The adoption of technological, administrative, and ancillar. Journal of Management, 13(4), 675-688. http://dx.doi.org/10.1177/014920638701300408.

Damanpour, F., \& Gopalakrishnan, S. (1998). Theories of organizational structure and innovation adoption: the role of environmental change. Journal of Engineering and Technology Management, 15(1), 1-24. http://dx.doi. org/10.1016/S0923-4748(97)00029-5.

Dewar, R. D., \& Dutton, J. E. (1986). The adoption of radical and incremental innovations: an empirical analysis. Management Science, 32(11), 132-155. http:// dx.doi.org/10.1287/mnsc.32.11.1422.

Earle, M. D. (1997). Changes in the food product development process. Trends in Food Science \& Technology, 8(1), 1924. http://dx.doi.org/10.1016/S0924-2244(96)20009-3.

Eklund, J. (2000). Development work for quality and ergonomics. Applied Ergonomics, 31(6), 641-648. http://dx.doi.org/10.1016/S0003-6870(00)00039-9. PMid:11132048.

Ettlie, J. E., Bridges, W. P., \& O'Keefe, R. D. (1984). Organization strategy and structural differences for radical versus incremental innovation. Management Science, 30(6), 682-695. http://dx.doi.org/10.1287/ mnsc.30.6.682.

Fàbregas-Fernández, A., García-Montoya, F., Pérez-Lozano, P., Suñé-Negre, J. M., Ticó, J. R., \& Miñarro, M. (2010). Quality assurance in research: incorporating ISSO 9001:2000 into a GMP quality management system in a pharmaceutical $\mathrm{R}+\mathrm{D}+\mathrm{I}$ Center. Accreditation and Quality Assurance, 15(5), 297-304. http://dx.doi. org/10.1007/s00769-009-0610-z.

Fryer, K. J., Antony, J., \& Douglas, A. (2007). Critical success factors of continuous improvement in the public sector. The TQM Magazine, 19(5), 497-517. http:// dx.doi.org/10.1108/09544780710817900.

Garcia, R., \& Calantone, R. (2002). A critical look at technological innovation typology and innovativeness terminology: a literature review. The Journal of Product Management, 19(2), 110-132. http://dx.doi.org/10.1016/ S0737-6782(01)00132-1.

Garcia-Sabater, J. J., Marin-Garcia, J. A., \& PerelloMarin, M. R. (2012). Is Implementation of Continuous Improvement Possible? An Evolutionary Model of Enablers and Inhibitors. Human Factors and Ergonomics in Manufacturing \& Service Industries, 22(2), 99-112. http://dx.doi.org/10.1002/hfm.20262.

Grover, V., Purvis, R. L., \& Segars, A. H. (2007). Exploring ambidextrous innovation tendencies in the adoption of telecommunications technologies. IEE Transactions on Engineering Management, 54(2), 268-285. http:// dx.doi.org/10.1109/TEM.2007.893995. 
He, Z., Cui, Q., \& He, S. (2006). Development of integrated quality information system for continuous improvement. In Proceedings of the IEEE International Conference on Management of Innovation and Technology (pp. 830-833). IEEE.

Henderson, R., \& Clark, K. B. (1990). Architectural Innovation: the reconfiguration of existing products technologies and the failure of established firms. Administrative Science Quarterly, 35(1), 9-30. http:// dx.doi.org/10.2307/2393549.

Hoerl, R. W., \& Gardner, M. M. (2010). Lean Six Sigma, creativity, and innovation. International Journal of Lean Six Sigma, 1(1), 30-38. http://dx.doi. org/10.1108/20401461011033149.

Imai, M. (1992). Kaizen: a estratégia para o sucesso competitivo. São Paulo: IMAM.

Irani, Z., \& Sharp, J. M. (1997). Integrating continuous improvement and innovation into a corporate culture: a case study. Technovation, 17(4), 199-206. http://dx.doi. org/10.1016/S0166-4972(96)00103-4.

Irani, Z., Beskese, A., \& Love, P. E. D. (2004). Total quality management and corporate culture: constructs of organizational excellence. Technovation, 24(8), 643650. http://dx.doi.org/10.1016/S0166-4972(02)00128-1.

Irani, Z., Sharp, J. M., \& Kagioglou, M. (1997). Improving business performance through developing a corporate culture. The TQM Magazine, 9(4), 206-216. http:// dx.doi.org/10.1108/09544789710168993.

Jager, B., Minnie, C., Jager, J., Welgemoed, M., Bessant, J., \& Francis, D. (2004). Enabling continuous improvement: a case study of implementation. Journal of Manufacturing Technology Management, 15(4), 315-324. http://dx.doi. org/10.1108/17410380410535017.

Jha, S., Noori, H., \& Michela, J. L. (1996). The dynamics of continuous improvement: Aligning organizational attributes and activities or quality and productivity. International Journal of Quality Science, 1(1), 19-47. http://dx.doi.org/10.1108/13598539610117975.

Kim, D., Kumar, V., \& Kumar, U. (2012). Relationship between quality management practices and innovation. Journal of Operations Management, 30(4), 295-315. http://dx.doi.org/10.1016/j.jom.2012.02.003.

Kitchenham, B. A., \& Charters, S. (2007). Guidelines for performing systematic literature reviews in software engineering. Keele University. Tech. Rep. EBSE-2007-01.

Kocaoglu, D. F., Deckro, R. F., Olson, M., Iyigun, G., Klein, J., \& Zhou, S. (1991). Framework for continuous improvement in new product development. In Proceedings of the Technology Management (pp. 294). IEEE.

Kowang, T. O., \& Rasli, A. (2011). New product development in multi-location R\&D organization: a concurrent engineering approach. African Journal of Business Management, 5(6), 2264-2275.
Lam, A. (2004). Organizational innovation. In, J. Fangerberg, D. Mowery \& R. R. Nelson. Handbook of innovation. Oxford University.

Lee, G., Bennett, D., \& Oakes, I. (2000). Technological and organisational change in small- to medium-sized manufacturing companies: A learning organisation perspective. International Journal of Operations \& Production Management, 20(5), 549-572. http://dx.doi. org/10.1108/01443570010318922.

Leifer, R., McDermott, C. M., O’Connor, G. C., Peters, M., \& Veryzer, R. W. (2000). Radical innovation: how mature companies can outsmart upstarts. Boston: Harvard Business School Press. 261 p.

Levy, Y., \& Ellis, T. (2006). A systems approach to conduct an effective literature review in support of information systems research. Informing Science, 9, 181-212.

Macdonald, J. (1995). Together TQM and BPR are winners. The TQM Magazine, 7(3), 21-25. http://dx.doi. org/10.1108/09544789510087706.

Marin-Garcia, J. A., Bautista, Y., Garcia-Sabater, J. J., \& Vidal-Carreras, P. I. (2010). Implantación de la innovación continua en la gestión de operaciones: una revisión de la literatura. Revista Innovar, 20(38), 77-94.

Martínez-Costa, M., \& Martínez-Lorente, A. R. (2008). Does quality management foster or hinder innovation? An empirical study of Spanish companies. Total Quality Management, 19(3), 209-221. http://dx.doi. org/10.1080/14783360701600639.

Martini, A., Laugen, B. T., Gastaldi, L., \& Corso, M. (2013). Continuous innovation: towards a paradoxical, ambidextrous combination of exploration and exploitation. International Journal of Technology Management, 61(1), 1-22. http://dx.doi.org/10.1504/IJTM.2013.050246.

Martowidjojo, A., \& Alamsjah, F. (2011). The role of organizational learning on innovation value chain. Proceedings of the IEEE International Conference on Management of Innovation and Technology (pp. 113-116). IEEE.

McAdam, R., \& Armstrong, G. (2001). A symbiosis of quality and innovation in SMEs: a multiple case study analysis. Managerial Auditing Journal, 16(7), 394-399. http://dx.doi.org/10.1108/02686900110398296.

McAdam, R., Armstrong, G., \& Kelly, B. (1998). Investigation of the relationship between total quality and innovation: a research study involving small organizations. European Journal of Innovation Management, 1(3), 139-147. http://dx.doi.org/10.1108/14601069810230216.

Mcadam, R., Moffett, S., Hazlett, S. A., \& Shevlin, M. (2010). Developing a model of innovation implementation for UK SMEs: A path analysis and explanatory case analysis. International Small Business Journal, 28(3), 195-214. http://dx.doi.org/10.1177/0266242609360610.

Mclaughlin, P., Bessant, J., \& Smart, P. (2008). Developing an organisation culture to facilitate radical innovation. 
International Journal of Technology Management, 44(3), 298. http://dx.doi.org/10.1504/IJTM.2008.021041.

Mesquita, M., \& Alliprandini, D. H. (2003). Competências essenciais para melhoria contínua da produção: Estudo de caso em empresas da indústria de autopeças. Revista Gestão \& Produção, 10(1), 17-33.

Middel, R., Weegh, S., \& Gieskes, J. (2007). Continuous improvement in The Netherlands: a survey-based study into current practices. International Journal of Technology Management, 37(3-4), 259-271. http:// dx.doi.org/10.1504/IJTM.2007.012262.

Mirica, M. P., \& Ito, N. C. (2010). Administração de P\&D na indústria de alta tecnologia: como gerenciar um paradoxo? In Anais do 13 SemeAd. São Paulo: SemeAD.

Naveh, E., \& Erez, M. (2004). Innovation and Attention to Detail in the Quality Improvement Paradigm. Management Science, 50(11), 1576-1586. http://dx.doi.org/10.1287/ mnsc. 1040.0272 .

Nijhof, A., Krabbendam, K., \& Looise, J. C. (2002). Innovation through exemptions: building upon the existing creativity of employees. Technovation, 22(11), 675-683. http://dx.doi.org/10.1016/S0166-4972(01)00088-8.

Nilsson-Witell, L., Antoni, M., \& Dahlgaard, J. J. (2005). Continuous improvement in product development: Improvement programs and quality principles. International Journal of Quality \& Reliability Management, 22(8), 754-776. http://dx.doi.org/10.1108/02656710510617210.

O'Brien, L., \& O'Reilly, P. (2010). An investigation of process innovation activity levels and their influencing factors in two irish manufacturing facilities. In Proceedings of the 2010 IEEE (pp. 863-868). IEEE.

Oliveira, M. A., Possamai, O., Valentina, L. V. O. D., \& Flesch, C. A. (2012). Applying Bayesian networks to performance forecast of innovation projects: A case study of transformational leadership influence in organizations oriented by projects. Expert Systems with Applications, 39(5), 5061-5070. http://dx.doi. org/10.1016/j.eswa.2011.11.033.

Oprime, P. C., \& Lizarelli, F. L. (2010). Relação entre estrutura para a melhoria contínua e desempenho e estrutura organizacional. Revista Produção Online, $10(2), 250-273$.

Organização para Cooperação e Desenvolvimento Econômico - OCDE. (2005). Manual de Oslo: diretrizes para coleta e interpretação de dados sobre inovação. Brasília.

Oxford University. Oxford dictionaries. Oxford University Press. Recuperado em fevereiro de 2013, de http:// english.oxforddictionaries.com

Peças, P., \& Jorge, A., Morgado, J., Henriques, E., \& Cernadas, R. (2012). Collaborative approach for performance improvement of non-added value activities in SMEs. In Proceedings of the 2012 18th International Conference on Engineering, Technology and Innovation (pp. 1-10)..
Perdomo-Ortiz, J., González-Benito, J., \& Galende, J. (2006). Total quality management as a forerunner of business innovation capability. Technovation, 26(10), 1170-1185. http://dx.doi.org/10.1016/j.technovation.2005.09.008.

Prajogo, D. I., \& Hong, S. W. (2008). The effect of TQM on performance in R\&D environments: a perspective from South Korean firms. Technovation, 28(12), 855-863. http://dx.doi.org/10.1016/j.technovation.2008.06.001.

Prajogo, D. I., \& Sohal, A. S. (2001). TQM and innovation: a literature review and research framework. Technovation, 21(9), 539-558. http://dx.doi.org/10.1016/S01664972(00)00070-5.

Regan, P., \& Kleiner, B. H. (1997). New developments in improving quality management in research and development. Aircraft Engineering and Aerospace Technology, 69(1), 26-30. http://dx.doi.org/10.1108/00022669710161621.

Rejeb, H. B., Morel-Guimarães, L., Boly, V., \& Assiélou, N. D. G. (2008). ASSIÉLOU, N'D. G. Measuring innovation best practices: improvement of an innovation index integrating threshold and synergy effects. Technovation, 28(12), 838-854. http://dx.doi. org/10.1016/j.technovation.2008.08.005.

Rossi, M., Taisch, M., \& Terzi, S. (2012). Lean Product Development: a five-steps methodology for continuous improvement. In Proceedings of the 2012 18th International Conference on Engineering, Technology and Innovation (pp. 1-10).

Sandoval-Arzaga, F., \& Suárez-Barraza, M. F. (2010). Experts within kaizen teams: how to get the most from their knowledge. Development and Learning Organizations, 24(4), 10-13. http://dx.doi.org/10.1108/14777281011056703.

Savolainen, T. I. (1999). Cycles of continuous improvement Realizing competitive advantages through quality. International Journal of Operations \& Production Management, 19(11), 1203-1222. http://dx.doi. org/10.1108/01443579910291096.

Schumpeter, J. (1985). A teoria do desenvolvimento econômico. Rio de Janeiro: Nova Cultural.

Sciulli, L. M. (1998). How organizational structure influences success in various types of innovation. Journal of Retail Banking Services, 20(1), 13-18.

Sohal, A. S., Terziovski, M., \& Zutshi, A. (2003). Teambased strategy at Varian Australia: a case study. Technovation, 23(4), 349-357. http://dx.doi.org/10.1016/ S0166-4972(01)00114-6.

Sopelana, A., Flores, M., Martinez, L., Flores, K., \& Sorli, M. (2012). The application of an assessment tool for lean product development: an exploratory study in spanish companies. In Proceedings of the 2012 18th International Conference on Engineering, Technology and Innovation (pp. 1-10).

Suárez-Barraza, M. F., \& Smith, T. (2014). The Kaizen approach within process innovation: findings from a multiple case study in Ibero-American countries. Total 
Quality Management\& Bussiness Excelence, 25(9-10), 1002-1025.

Sun, H., Zhao, Y., \& Yau, H. K. (2009). The relationship between quality management and the speed of new product development. The TQM Journal, 21(6), 576588. http://dx.doi.org/10.1108/17542730910995855.

Terziovski, M. (2002). Achieving performance excellence through an integrated strategy of radical innovation and continuous improvement. Measuring Business Excellence, 6(2), 5-14. http://dx.doi.org/10.1108/13683040210431419.

Terziovski, M., \& Sohal, A. S. (2000). The adoption of continuous improvement and innovation strategies in Australian manufacturing firms. Technovation, 20(10), 539-550. http://dx.doi.org/10.1016/S01664972(99)00173-X.

Thamhain, H. J. (2003). Managing innovative R\&D teams. $R \& D$ Management, 33(3), 297-311. http://dx.doi. org/10.1111/1467-9310.00299.

Tidd, J., Bessant, J., \& Pavitt, K. (2008). Gestão da inovação (3. ed.). Porto Alegre: Bookman. 600 p.

Toledo, J. C. (1994). Gestão da mudança da qualidade do produto. Revista Gestão \& Produção, 1(2), 104-125.

Tonnessen, T. (2005). Continuous innovation through company wide employee participation. The TQM Magazine, 17(2), 195-207. http://dx.doi.org/10.1108/09544780510583254.

Tranfield, D., Denyer, D., \& Smart, P. (2003). Towards a methodology for developing evidence-informed management knowledge by means of systematic review.
British Journal of Management, 14(3), 207-222. http:// dx.doi.org/10.1111/1467-8551.00375.

Tushman, M. L., \& O’Reilly, C. A. 3rd (1996). Organizations: managing evolutionary and revolutionary change. California Management Review, 38(4), 8-30. http:// dx.doi.org/10.2307/41165852.

Wordnet ${ }^{\circledR}$. Pinceton University. Recuperado em fevereiro de 2013, de http://wordnetweb.princeton.edu/perl/webwn

Yan, B., \& Makinde, O. D. (2011). Impact of continuous improvement on new product development within SMEs in the Western Cape, South Africa. African Journal of Business Management, 5(6), 2220-2229.

Yan, B., \& Makinde, O. D. (2009). Modelling the long term impact of existing products on perceived value of new products. In Proceedings of the 2009 IEEE, IEEM - Industrial Engineering and Engineering Management (pp. 1136-1140). IEEE.

Yang, C. L., Lin, S. P., Chan, Y., \& Sheu, C. (2010). Mediated effect of environmental management on manufacturing competitiveness: an empirical study. International Journal of Production Economics, 123(1), 210-220. http://dx.doi.org/10.1016/j.ijpe.2009.08.017.

Zairi, M. (1995). Moving from continuous to discontinuous innovation in FMCG: a re-engineering perspective. World Class Design to Manufacture, 2(5), 32-37. http:// dx.doi.org/10.1108/09642369310095201.

Zampini, C. S., \& Toledo, J. C. (2010). Proposta para estruturação da gestão da melhoria contínua em uma fabricante de bebidas. Gepros. Gestão da Produção, Operações e Sistemas, 5(2), 107-138. 